\title{
Tight Frame with Hahn and Krawtchouk Polynomials of Several Variables
}

Yuan $X U$

Department of Mathematics, University of Oregon, Eugene, Oregon 97403-1222, USA

E-mail:yuan@uoregon.edu

URL: http://pages.uoregon.edu/yuan/

Received November 06, 2013, in final form February 25, 2014; Published online March 03, 2014

http://dx.doi.org/10.3842/SIGMA.2014.019

\begin{abstract}
Finite tight frames for polynomial subspaces are constructed using monic Hahn polynomials and Krawtchouk polynomials of several variables. Based on these polynomial frames, two methods for constructing tight frames for the Euclidean spaces are designed. With $\mathrm{r}(d, n):=\left(\begin{array}{c}n+d-1 \\ n\end{array}\right)$, the first method generates, for each $m \geq n$, two families of tight frames in $\mathbb{R}^{r(d, n)}$ with $r(d+1, m)$ elements. The second method generates a tight frame in $\mathbb{R}^{r(d, N)}$ with $1+N \times \mathrm{r}(d+1, N)$ vectors. All frame elements are given in explicit formulas.

Key words: Jacobi polynomials; simplex; Hahn polynomials; Krawtchouk polynomials; several variables; tight frame
\end{abstract}

2010 Mathematics Subject Classification: 33C50; 42C15

\section{Introduction}

For a real finite dimension space $V$ with inner product $(\cdot, \cdot)$, a set of vectors $\left\{v_{k}: k=1, \ldots, M\right\}$ in $V$ is called a (Parseval) tight frame with $M$ elements if

$$
(v, v)=\sum_{k=1}^{M}\left(v, v_{k}\right)^{2}, \quad \forall v \in V .
$$

Tight frames are connected to a wide variety of problems in pure and applied mathematics and they have been studied intensively in recent years (see, for examples, [1, 2, 3, 4, 9] and references therein). We construct tight frames with the Hahn and Krawtchouk polynomials of several variables, and use them to construct several families of tight frames for the Euclidean spaces that are given in explicit formulas.

Let $\Pi_{n}^{d}$ denote the space of polynomials of total degree at most $n$ in $d$-variables. Let $\langle\cdot, \cdot\rangle$ denote an inner product defined on the space of polynomials or on $\Pi_{N}^{d}$ for a fixed integer $N$. A polynomial $p \in \Pi_{n}^{d}$ is called an orthogonal polynomial of degree $n$ with respect to the inner product if

$$
\langle p, q\rangle=0 \quad \text { for all } \quad q \in \Pi_{n-1}^{d} .
$$

Let $\mathcal{V}_{n}^{d}$ denote the space of orthogonal polynomials of degree $n$. Then

$$
\operatorname{dim} \mathcal{V}_{n}^{d}=\left(\begin{array}{c}
n+d-1 \\
n
\end{array}\right), \quad n=0,1,2, \ldots
$$

For $\nu \in \mathbb{N}_{0}^{d}$, let $|\nu|:=\nu_{1}+\cdots+\nu_{d}$. A basis $\left\{P_{\nu}:|\nu|=n\right\}$ of $\mathcal{V}_{n}^{d}$ is called mutually orthogonal if $\left\langle P_{\nu}, P_{\mu}\right\rangle=0$ whenever $\nu \neq \mu$ and $|\nu|=|\mu|=n$, and it is called orthonormal if, in addition, $\left\langle P_{\nu}, P_{\nu}\right\rangle=1$. The space $\mathcal{V}_{n}^{d}$ can have many distinct bases. 
For $a \in \mathbb{R}$ and $n \in \mathbb{N}_{0}^{d}$, let $(a)_{n}=a(a+1) \cdots(a+n-1)$ be the Pochhammer symbol. For $x \in \mathbb{R}^{d}$ and $\nu \in \mathbb{N}_{0}^{d}$ we let $x^{\nu}:=x_{1}^{\nu_{1}} \cdots x_{d}^{\nu_{d}}$, and let

$$
\nu !:=\nu_{1} ! \cdots \nu_{d} ! \quad \text { and } \quad(\nu)_{\mu}:=\left(\nu_{1}\right)_{\mu_{1}} \cdots\left(\nu_{d}\right)_{\mu_{d}} .
$$

The Hahn polynomials and Krawtchouk polynomials of $d$-variables are discrete orthogonal polynomials defined on the homogeneous coordinates of

$$
\mathbb{Z}_{N}^{d+1}:=\left\{\alpha \in \mathbb{Z}^{d+1}:|\alpha|=\alpha_{1}+\cdots+\alpha_{d+1}=N\right\}
$$

For $\kappa \in \mathbb{R}^{d+1}$ with $\kappa_{1}>-1, \ldots, \kappa_{d+1}>-1$, the Hahn polynomials are orthogonal with respect to the inner product

$$
\langle f, g\rangle_{\mathrm{H}_{\kappa, N}}:=\frac{N !}{(|\kappa|+d+1)_{N}} \sum_{x \in Z_{N}^{d+1}} f(x) g(x) \mathrm{H}_{\kappa}(x), \quad \mathrm{H}_{\kappa}(x):=\frac{(\kappa+\mathbf{1})_{\alpha}}{\alpha !}
$$

where $1:=(1, \ldots, 1)$. For $\rho \in \mathbb{R}^{d}$ with $0<\rho_{1}, \ldots, \rho_{d}<1$ and $|\rho|<1$, the Krawtchouk polynomials are orthogonal with respect to the inner product

$$
\langle f, g\rangle_{\mathrm{K}_{\rho, N}}:=\frac{1}{N !} \sum_{x \in Z_{N}^{d+1}} f(x) g(x) \mathrm{K}_{\rho}(x), \quad \mathrm{K}_{\rho}(x):=\frac{\boldsymbol{\rho}^{\kappa}}{x !},
$$

where $\boldsymbol{\rho}=(\rho, 1-|\rho|)$. These polynomials have been studied extensively in the literature. For this paper, the most relevant work on the Hahn polynomials are those in $[7,8,10,11,12,13$, 14, 15, 19]. For the Krawtchouk polynomials, we refer to [5, 14, 19] and references therein. In both cases, a family of mutually orthogonal polynomials can be defined in terms of the classical Hahn polynomials or Krawtchouk polynomials of one variable, and they have been used in most of the studies in the literature.

In the present paper, we study monic Hahn and Krawtchouk polynomials instead. These are orthogonal projections of $(-1)^{|\alpha|}(-x)_{\alpha}$ on either $\mathcal{V}_{n}^{d}\left(\mathrm{H}_{\kappa, N}\right)$ or $\mathcal{V}_{n}^{d}\left(\mathrm{~K}_{\rho, N}\right)$. For $\alpha \in \mathbb{Z}_{m}^{d+1}$ and $m \geq n$, let $\mathrm{U}_{\alpha, n}(x)=(-1)^{|\alpha|}(-x)_{\alpha}+\cdots$ denote the monic orthogonal polynomial of degree $n$. Then $\Xi_{n, m}:=\left\{\bigcup_{\alpha, n}: \alpha \in \mathbb{Z}_{m}^{d+1}\right\}$ is a subset of $\mathcal{V}_{n}^{d}$ and it contains an orthogonal basis of $\mathcal{V}_{n}^{d}$. In the special case of $m=n$ and $\alpha=\left(\alpha^{\prime}, n-\left|\alpha^{\prime}\right|\right)$ with $\alpha^{\prime} \in \mathbb{N}_{0}^{d}$ and $\left|\alpha^{\prime}\right| \leq n$, the monic Hahn and Krawtchouk polynomials were studied in [13, 14]. We shall describe generating functions, connection to the mutually orthogonal polynomials, and explicit expressions for these polynomials. Furthermore, the set $\Xi_{n, m}$ contains far more elements than the dimension of $\mathcal{V}_{n}^{d}$, we shall prove that the set $\Xi_{n, m}$ is a tight frame for the space $\mathcal{V}_{n}^{d}$ and these frames are given in explicit formulas. The connection between tight frames and orthogonal polynomials of several variables has been explored for the Jacobi polynomials on the simplex [16] (see also [17, 19]). The discrete orthogonal polynomials have not been used in this connection until now.

A finite tight frame is equivalent to a tight frame on an Euclidean space. From our Hahn and Krawtchouk polynomial frames, we are able to derive a large family of tight frames for Euclidean spaces that are given by explicit formulas. There are many ways of constructing tight frames for the Euclidean spaces. One can, for example, use the classical discrete Fourier transform to construct tight frame for $\mathbb{C}^{n}$ or $\mathbb{R}^{n}$ that contains $m$ elements for all $m>n$. There are also algorithms that can be used to construct tight frames with certain additional features [3]. Our frames are all given by explicit formulas. In fact, we shall give two methods for constructing such frames. For $n, d \in \mathbb{N}$, let

$$
\mathrm{r}(d, n):=\left(\begin{array}{c}
n+d-1 \\
n
\end{array}\right)=\operatorname{dim} \mathcal{V}_{n}^{d} \quad \text { and } \quad \mathrm{n}(d, n):=\mathrm{r}(d+1, n) .
$$


For a positive integer $m \geq n$, our first method uses either the Hahn polynomial frames or the Krawtchouk polynomial frames to construct explicitly tight frames in $\mathbb{R}^{\mathrm{n}(d, n)}$ that contain $\mathrm{n}(d, m)$ elements. Our second method uses the Hahn polynomial frames with the parameter $\kappa=0$ to construct tight frames in $\mathbb{R}^{\mathrm{n}(d, N)}$ with $N \times \mathrm{n}(d, N)+1$ elements. All frames are given by explicit formulas and those derived from the second method have the additional feature that all but one frame elements are vectors whose entries consist of only rational numbers.

The paper is organized as follows. In the next section, we recall necessary background on orthogonal polynomials. Monic Hahn polynomials are introduced and developed in Section 3, from which monic Krawtchouk polynomials are deduced by taking an appropriate limit in Section 4. Tight frames for the Hahn and the Krawtchouk polynomials, as well as for the Euclidean spaces are discussed in Section 5, where several examples are explicitly given.

\section{Background and preliminary}

Throughout this paper we reserve the Greek letter $\kappa$ and $\rho$ for the parameters in the weight function $\mathrm{H}_{\kappa, N}$ and $\mathrm{K}_{\rho}$. We shall use other Greek letters for the multiindexes. Orthogonal polynomials in $\mathcal{V}_{n}^{d}$ are indexed by $\nu$ or $\mu$ in $\mathbb{N}_{0}^{d}$, and we use $\alpha, \beta, \gamma$ for indexes in $\mathbb{N}_{0}^{d+1}$.

For $a, b>-1$, the classical Hahn polynomial $Q_{n}(x ; a, b, N)$ is a ${ }_{3} F_{2}$ hypergeometric function given by

$$
\mathrm{Q}_{n}(x ; a, b, N):={ }_{3} F_{2}\left(\begin{array}{c}
-n, n+a+b+1,-x \\
a+1,-N
\end{array} ;\right), \quad n=0,1, \ldots, N .
$$

These are discrete orthogonal polynomials defined on the set $\{0,1, \ldots, N\}$. For the Hahn polynomials of $d$-variables, a mutually orthogonal basis for $\mathcal{V}_{n}^{d}\left(\mathrm{H}_{\kappa, N}\right)$ can be given in terms of $\mathrm{Q}_{n}(x ; a, b, N)$. We need the following definition.

For $y=\left(y_{1}, \ldots, y_{d}\right) \in \mathbb{R}^{d}$ and $1 \leq j \leq d$, we define

$$
\mathbf{y}_{j}:=\left(y_{1}, \ldots, y_{j}\right) \quad \text { and } \quad \mathbf{y}^{j}:=\left(y_{j}, \ldots, y_{d}\right) .
$$

We also define $\mathbf{y}_{0}:=\varnothing$ and $\mathbf{y}^{d+1}:=\varnothing$. In particular, $\mathbf{y}_{d}=\mathbf{y}^{1}=y$ and

$$
\left|\mathbf{y}_{j}\right|=y_{1}+\cdots+y_{j}, \quad\left|\mathbf{y}^{j}\right|=y_{j}+\cdots+y_{d}, \quad \text { and } \quad\left|\mathbf{y}_{0}\right|=\left|\mathbf{y}^{d+1}\right|=0 \text {. }
$$

For the parameter vector $\kappa=\left(\kappa_{1}, \ldots, \kappa_{d+1}\right)$, we defined $\kappa^{j}:=\left(\kappa_{j}, \ldots, \kappa_{d+1}\right)$ for $1 \leq j \leq d+1$. For $\nu \in \mathbb{N}_{0}^{d}$ and $x \in \mathbb{R}^{d}$, define

$$
a_{j}:=a_{j}(\kappa, \nu):=\left|\kappa^{j+1}\right|+2\left|\nu^{j+1}\right|+d-j, \quad 1 \leq j \leq d .
$$

Proposition 2.1. For $x \in \mathbb{Z}_{N}^{d+1}$ and $\nu \in \mathbb{N}_{0}^{d},|\nu| \leq N$, define

$$
\begin{aligned}
\mathrm{H}_{\nu}(x ; \kappa, N):= & \frac{(-1)^{|\nu|}}{(-N)_{|\nu|}} \prod_{j=1}^{d} \frac{\left(\kappa_{j}+1\right)_{\nu_{j}}}{\left(a_{j}+1\right)_{\nu_{j}}}\left(-N+\left|\mathbf{x}_{j-1}\right|+\left|\nu^{j+1}\right|\right)_{\nu_{j}} \\
& \times \mathrm{Q}_{\nu_{j}}\left(x_{j} ; \kappa_{j}, a_{j},-N+\left|\mathbf{x}_{j-1}\right|-\left|\nu^{j+1}\right|\right) .
\end{aligned}
$$

Then the polynomials in $\left\{\mathrm{H}_{\nu}(x ; \kappa, N):|\nu|=n\right\}$ form a mutually orthogonal basis of $\mathcal{V}_{n}^{d}\left(\mathrm{H}_{\kappa, N}\right)$ and $\mathrm{B}_{\nu}:=\left\langle\mathrm{H}_{\nu}(\cdot ; \kappa, N), \mathrm{H}_{\nu}(\cdot ; \kappa, N)\right\rangle_{\mathrm{H}_{\kappa, N}}$ is given by, setting $\lambda_{\kappa}:=|\kappa|+d+1$,

$$
\mathrm{B}_{\nu}(\kappa, N):=\frac{(-1)^{|\nu|}\left(\lambda_{k}\right)_{N+|\nu|}}{(-N)_{|\nu|}\left(\lambda_{k}\right)_{N}\left(\lambda_{k}\right)_{2|\nu|}} \prod_{j=1}^{d} \frac{\left(\kappa_{j}+a_{j}+1\right)_{2 \nu_{j}}\left(\kappa_{j}+1\right)_{\nu_{j}} \nu_{j} !}{\left(\kappa_{j}+a_{j}+1\right)_{\nu_{j}}\left(a_{j}+1\right)_{\nu_{j}}} .
$$


This basis was defined in [11] and given in the present form in [10, 14, 18]. Another basis for $\mathcal{V}_{n}^{d}\left(\mathrm{H}_{\kappa, N}\right)$ that is of interests for our work is the monic orthogonal basis studied in [13], which will come out as a special case of the discussion in the next section. It is worth mentioning that, for $\nu \in \mathbb{N}_{0}^{d}, m, N=0,1, \ldots$,

$$
\frac{\mathrm{B}_{\nu}(\kappa, N)}{\mathrm{B}_{\nu}(\kappa, m)}=\frac{(|\kappa|+d+1)_{N+|\nu|}(|\kappa|+d+1)_{m}(-m)_{|\nu|}}{(|\kappa|+d+1)_{m+|\nu|}(|\kappa|+d+1)_{N}(-N)_{|\nu|}}
$$

depends only on $|\nu|$, not the elements of $\nu$. The expansion of $\mathrm{H}_{\nu}(x ; \kappa, N)$ in the shifted monic monomials $(-x)_{\alpha}$ is given in [19].

Proposition 2.2. For $\nu \in \mathbb{N}_{0}^{d}$ with $|\nu|=n$ and $x \in \mathbb{Z}_{N}^{d+1}$,

$$
\mathrm{H}_{\nu}(x ; \kappa, N)=\frac{n !}{(-N)_{n}} \sum_{|\alpha|=n} \frac{\mathrm{H}_{\nu}(\alpha ; \kappa, n)}{\alpha !}(-x)_{\alpha} .
$$

There is a close relation between the Hahn polynomials and the orthogonal polynomials with respect to the weight function

$$
W_{\kappa}(x):=x_{1}^{\kappa_{1}} \cdots x_{d}^{\kappa_{d}}(1-|x|)^{\kappa_{d+1}}, \quad \kappa_{1}>-1, \ldots, \kappa_{d+1}>-1,
$$

on the simplex $T^{d}:=\left\{x \in \mathbb{R}^{d}: x_{1} \geq 0, \ldots, x_{d} \geq 0,|x| \leq 1\right\}$. Let

$$
\langle f, g\rangle_{W_{\kappa}}:=w_{\kappa} \int_{T^{d}} f(x) g(x) W_{\kappa}(x) d x \quad \text { with } \quad w_{\kappa}:=\frac{\Gamma(|\kappa|+d+1)}{\prod_{i=1}^{d+1} \Gamma\left(\kappa_{i}+1\right)}
$$

A mutually orthogonal basis for $\mathcal{V}_{n}^{d}\left(W_{\kappa}\right)$ can be given in terms of the classical Jacboi polynomials

$$
\frac{P_{n}^{(a, b)}(t)}{P_{n}^{(a, b)}(1)}={ }_{2} F_{1}\left(-n, n+a+b+1 ; a+1 ; \frac{1-t}{2}\right) .
$$

Proposition 2.3. For $\nu \in \mathbb{N}_{0}^{d}$ and $x \in \mathbb{R}^{d}$, define

$$
P_{\nu}^{\kappa}(x):=\prod_{j=1}^{d}\left(\frac{1-\left|\mathbf{x}_{j}\right|}{1-\left|\mathbf{x}_{j-1}\right|}\right)^{\left|\nu^{j+1}\right|} \frac{P_{\nu_{j}}^{\left(a_{j}, \kappa_{j}\right)}\left(\frac{2 x_{j}}{1-\left|\mathbf{x}_{j-1}\right|}-1\right)}{P_{\nu_{j}}^{\left(a_{j}, \kappa_{j}\right)}(1)},
$$

where $a_{j}$ is defined by (2.3). Then the polynomials in $\left\{P_{\nu}^{\kappa}:|\nu|=n\right\}$ form a mutually orthogonal basis of $\mathcal{V}_{n}^{d}\left(W_{\kappa}\right)$ with $\left\langle P_{\nu}^{\kappa}, P_{\nu}^{\kappa}\right\rangle_{W_{\kappa}}$ given by

$$
\left\langle P_{\nu}^{\kappa}, P_{\nu}^{\kappa}\right\rangle_{W_{\kappa}}=\frac{1}{(|\kappa|+d+1)_{2|\nu|}} \prod_{j=1}^{d} \frac{\left(\kappa_{j}+a_{j}+1\right)_{2 \nu_{j}}\left(\kappa_{j}+1\right)_{\nu_{j}} \nu_{j} !}{\left(\kappa_{j}+a_{j}+1\right)_{\nu_{j}}\left(a_{j}+1\right)_{\nu_{j}}} .
$$

This basis is well studied, but our normalization is different from the usual definition (see [6, p. 47]) by $P_{\nu_{j}}^{\left(a_{j}, \kappa_{j}\right)}(1)$ in the denominator. As defined in [11] and later recognized in [18], the polynomials $P_{\nu}^{\kappa}$ serve as generating function for the Hahn polynomials.

Proposition 2.4. Let $\kappa \in \mathbb{R}^{d+1}$ with $\kappa_{i}>-1$ and $N \in \mathbb{N}$. For $\nu \in \mathbb{N}_{0}^{d},|\nu| \leq N$, and $y=\left(y^{\prime}, y_{d+1}\right) \in \mathbb{R}^{d+1}$,

$$
|y|^{N} P_{\nu}^{\kappa}\left(\frac{y^{\prime}}{|y|}\right)=\sum_{\alpha \in \mathbb{Z}_{N}^{d+1}} \frac{N !}{\alpha !} \mathrm{H}_{\nu}(\alpha ; \kappa, N) y^{\alpha} .
$$


The Hahn polynomials also appear as connecting coefficients between $P_{\nu}^{\kappa}$ and monic orthogonal polynomials $R_{\alpha}^{\kappa}$ defined by, for $\alpha \in \mathbb{N}_{0}^{d+1}$ and $x \in \mathbb{R}^{d}$,

$$
R_{\alpha}^{\kappa}(x):=X^{\alpha}+q_{\alpha}(x), \quad q \in \Pi_{|\alpha|-1}^{d}, \quad \text { where } \quad X=(x, 1-|x|) .
$$

The explicit formula of $R_{\alpha}(x)$ was derived in [18].

Theorem 2.5. For $\alpha \in \mathbb{N}_{0}^{d+1}$ and $x \in \mathbb{R}^{d}$,

$$
R_{\alpha}^{\kappa}(x)=\frac{(-1)^{|\alpha|}(\kappa+\mathbf{1})_{\alpha}}{(|\kappa|+d+|\alpha|)_{|\alpha|}} \sum_{\gamma \leq \alpha} \frac{(-\alpha)_{\gamma}(|\kappa|+d+|\alpha|)_{|\gamma|}}{(\kappa+\mathbf{1})_{\gamma} \gamma !} X^{\gamma} .
$$

Furthermore, $\left\{R_{\alpha}^{\kappa}:|\alpha|=n, \alpha_{d+1}=0\right\}$ is a basis of $\mathcal{V}_{n}^{d}\left(W_{\kappa}\right)$.

The cardinality of the set $\left\{R_{\alpha}^{\kappa}: \alpha \in \mathbb{N}_{0}^{d},|\alpha|=N\right\}$ is larger than the dimension of $\mathcal{V}_{n}^{d}\left(W_{\kappa}\right)$. The Hahn polynomials serve as the connecting coefficients of $P_{\nu}^{\kappa}$ and $R_{\alpha}^{k}$ as shown recently in [19].

Theorem 2.6. For $\nu \in \mathbb{N}_{0}^{d}$ with $|\nu|=n$ and $\alpha \in \mathbb{Z}_{n}^{d+1}$,

$$
P_{\nu}^{\kappa}(x)=\sum_{|\alpha|=n} \frac{n !}{\alpha !} \mathrm{H}_{\nu}(\alpha ; \kappa, n) R_{\alpha}^{\kappa}(x),
$$

and, conversely,

$$
R_{\alpha}^{\kappa}(x)=\frac{(\kappa+\mathbf{1})_{\alpha}}{(|\kappa|+d+1)_{n}} \sum_{|\nu|=n} \frac{\mathrm{H}_{\nu}(\alpha ; \kappa, n)}{\mathrm{B}_{\nu}(\kappa, n)} P_{\nu}^{\kappa}(x) .
$$

We now turn to basic results on the Krawtchouk polynomials. For $0<p<1$, the classical Krawtchouk polynomial $\mathrm{K}_{n}(x ; p, N)$ of one variable is defined by

$$
\mathrm{K}_{n}(x ; p, N):={ }_{2} F_{1}\left(\begin{array}{c}
-n,-x \\
-N
\end{array} ; \frac{1}{p}\right), \quad n=0,1, \ldots, N .
$$

For $\nu \in \mathbb{N}_{0}^{d}$, a family of the Krawtchouk polynomials of $d$ variables can be given in terms of Krawtchouk polynomials of one variable $[10,14]$. For $\rho \in \mathbb{N}_{0}^{d}$, we use the notation (2.2), which implies that $\left|\boldsymbol{\rho}_{j}\right|=\rho_{1}+\cdots+\rho_{j}$ for $j=1,2, \ldots, d$.

Proposition 2.7. Let $\rho \in \mathbb{R}^{d}$ with $0<\rho_{i}<1$ and $|\rho|<1$. For $\nu \in \mathbb{N}_{0}^{d},|\nu| \leq N$, and $x \in \mathbb{R}^{d}$, define

$$
\begin{aligned}
\mathrm{K}_{\nu}(x ; \rho, N):= & \frac{(-1)^{|\nu|}}{(-N)_{|\nu|}} \prod_{j=1}^{d} \frac{\rho_{j}^{\nu_{j}}}{\left(1-\left|\boldsymbol{\rho}_{j}\right|\right)^{\nu_{j}}}\left(-N+\left|\mathbf{x}_{j-1}\right|+\left|\nu^{j+1}\right|\right)_{\nu_{j}} \\
& \times \mathrm{K}_{\nu_{j}}\left(x_{j} ; \frac{\rho_{j}}{1-\left|\boldsymbol{\rho}_{j-1}\right|}, N-\left|\mathbf{x}_{j-1}\right|-\left|\nu^{j+1}\right|\right) .
\end{aligned}
$$

The polynomials in $\left\{\mathrm{K}_{\nu}(\cdot ; \rho, N):|\nu|=n\right\}$ form a mutually orthogonal basis of $\mathcal{V}_{n}^{d}\left(\mathrm{~K}_{\rho, N}\right)$ and $\mathrm{C}_{\nu}(\rho, N):=\left\langle\mathrm{K}_{\nu}(\cdot ; \rho, N), \mathrm{K}_{\nu}(\cdot ; \rho, N)\right\rangle_{\mathrm{K}_{\rho, N}}$ is given by

$$
\mathrm{C}_{\nu}(\rho, N):=\frac{(-1)^{|\nu|}}{(-N)_{|\nu|} N !} \prod_{j=1}^{d} \frac{\nu_{j} ! \rho_{j}^{\nu_{j}}}{\left(1-\left|\boldsymbol{\rho}_{j}\right|\right)^{\nu_{j}-\nu_{j+1}}} .
$$

The Krawtchouk polynomials in (2.10) are limits of the Hahn polynomials in (2.4). More precisely, setting $\kappa=t(\rho, 1-|\rho|)$, we have [10]

$$
\lim _{t \rightarrow \infty} \mathrm{H}_{\nu}(x ; t(\rho, 1-|\rho|), N)=\mathrm{K}_{\nu}(x ; \rho, N) .
$$




\section{Monic Hahn polynomials of several variables}

The monic polynomial $R_{\alpha}^{\kappa}$ has a single monomial $x^{\alpha}$ as its highest term. For the Hahn polynomials, the role of $x^{\alpha}$ is played by $\mathrm{m}_{\alpha}$ defined as follows.

Definition 3.1. For $x \in \mathbb{R}^{d+1}$ and $\alpha \in \mathbb{N}_{0}^{d+1}$, define

$$
\mathrm{m}_{\alpha}(x):=(-1)^{|\alpha|}(-x)_{\alpha}=x^{\alpha}+q_{\alpha}, \quad q_{\alpha} \in \Pi_{n-1}^{d+1} .
$$

It follows that $\mathrm{m}_{\alpha}(x)$ is a monic polynomial of degree $|\alpha|$. For each $\alpha \in \mathbb{Z}_{N}^{d+1}$, we define the monic Hahn polynomial as the orthogonal polynomial that has $\mathrm{m}_{\alpha}$ as its leading term.

Definition 3.2. For $\alpha \in \mathbb{N}_{0}^{d+1}$ and $|\alpha| \leq N$, the monic Hahn polynomial $Q_{\alpha}(\cdot ; \kappa, N)$ in $\mathcal{V}_{|\alpha|}^{d}\left(\mathrm{H}_{\kappa, N}\right)$ is defined uniquely by

$$
\mathrm{Q}_{\alpha}(x ; \kappa, N):=\mathrm{m}_{\alpha}(x)+q_{\alpha}(x), \quad q \in \Pi_{|\alpha|-1}^{d}, \quad x \in \mathbb{Z}_{N}^{d+1} .
$$

The polynomials $\mathrm{Q}_{\alpha}(\cdot ; \kappa, N)$ are discrete counterparts of monic orthogonal polynomial $R_{\alpha}^{\kappa}$. Like $R_{\alpha}$, the cardinality of the set $\left\{\mathrm{Q}_{\alpha}(\cdot ; \kappa, N):|\alpha|=n\right\} \in \mathcal{V}_{n}^{d}\left(H_{\kappa, N}\right)$ is much larger than the dimension of $\mathcal{V}_{n}^{d}\left(\mathrm{H}_{\kappa, N}\right)$, so that the set contains redundancy.

In parallel to the relations between the monic orthogonal polynomials $R_{\alpha}^{\kappa}$ and the Jacobi polynomials $P_{\nu}^{\kappa}$, in Theorem 2.6, we can derive relations between $\mathrm{Q}_{\beta}(\cdot ; \kappa, N)$ and the Hahn polynomials $\mathrm{H}_{\nu}(\cdot ; \kappa, N)$.

Proposition 3.3. For $x \in \mathbb{Z}_{N}^{d+1}, \alpha \in \mathbb{Z}_{n}^{d+1}$ and $\nu \in \mathbb{N}_{0}^{d}$ with $|\nu|=n$,

$$
\mathrm{H}_{\nu}(x ; \kappa, N)=\frac{(-1)^{n} n !}{(-N)_{n}} \sum_{|\alpha|=n} \frac{\mathrm{H}_{\nu}(\alpha ; \kappa, n)}{\alpha !} \mathrm{Q}_{\alpha}(x ; \kappa, N),
$$

and, conversely,

$$
\mathrm{Q}_{\alpha}(x ; \kappa, N)=\frac{(\kappa+\mathbf{1})_{\alpha}(-N)_{n}}{(-1)^{n}(|\kappa|+d+1)_{n}} \sum_{|\nu|=n} \frac{\mathrm{H}_{\nu}(\alpha ; \kappa, n)}{\mathrm{B}_{\nu}(\kappa, n)} \mathrm{H}_{\nu}(x ; \kappa, N) .
$$

Proof. Substituting $\mathrm{m}_{\beta}(x)=\mathrm{Q}_{\beta}(x)-q_{\beta}$ into (2.7), we see that (3.1) follows from the orthogonality of $\mathrm{H}_{\nu}(\cdot ; \kappa, N)$. Conversely, since $\left\{\mathrm{H}_{\nu}(\cdot ; \kappa, N):|\nu|=n\right\}$ is a basis of $\mathcal{V}_{n}^{d}\left(\mathrm{H}_{\kappa, N}\right)$, there are unique constants $c_{\beta, \nu}$ such that $\mathrm{H}_{\beta}(\cdot ; \kappa, N)=\sum_{|\nu|=n} c_{\beta, \nu} \mathrm{Q}_{\nu}(\cdot ; \kappa, N)$. Hence, by (3.1), we obtain

$$
\mathrm{H}_{\nu}(x ; \kappa, N)=\frac{(-1)^{n} n !}{(-N)_{n}} \sum_{|\mu|=n} \sum_{|\beta|=n} \frac{\mathrm{H}_{\nu}(\beta ; \kappa, n)}{\beta !} c_{\beta, \mu} \mathrm{H}_{\mu}(x ; \kappa, N) .
$$

Since $\mathrm{H}_{\nu}(\cdot, \kappa, N)$ are mutually orthogonal, we must have

$$
\frac{(-1)^{n} n !}{(-N)_{n}} \sum_{|\beta|=n} \frac{\mathrm{H}_{\nu}(\beta ; \kappa, n)}{\beta !} c_{\beta, \mu}=\delta_{\nu, \mu}
$$

The orthogonality of $\mathrm{H}_{\nu}(\cdot ; \kappa, n)$ gives one solution of $c_{\beta, \mu}$. The uniqueness of $c_{\beta, \mu}$ shows that it is the only solution and proves (3.2).

Throughout the rest of this section, we often use the abbreviation

$$
\lambda_{k}:=|\kappa|+d+1 .
$$

Recall that the Jacobi polynomials $P_{\nu}^{\kappa}$ are generating functions of the Hahn polynomials $\mathrm{H}_{\nu}(\cdot ; \kappa, N)$. It turns out that $R_{\alpha}^{\kappa}$ are generating functions of the monic orthogonal polynomials $\mathrm{Q}_{\nu}(\cdot ; \kappa, N)$. 
Theorem 3.4. Let $y=\left(y^{\prime}, y_{d+1}\right) \in \mathbb{R}^{d+1}$. For $\beta \in \mathbb{N}_{0}^{d+1}$ with $|\beta| \leq N$,

$$
R_{\beta, N}^{\kappa}(y):=|y|^{N} R_{\beta}^{\kappa}\left(\frac{y^{\prime}}{|y|}\right)=\frac{(-1)^{|\beta|}}{(-N)_{|\beta|}} \sum_{|\alpha|=N} \frac{N !}{\alpha !} \mathrm{Q}_{\beta}(\alpha ; \kappa, N) y^{\alpha} .
$$

Proof. Recall that $P_{\nu, N}^{\kappa}(y)=|y|^{N} P_{\nu}(y /|y|)$. Let $n=|\beta|$. It follows from (2.9), (2.8) and (3.2) that

$$
\begin{aligned}
R_{\beta, N}^{\kappa}(y) & =\frac{(\kappa+\mathbf{1})_{\beta}}{\left(\lambda_{\kappa}\right)_{n}} \sum_{|\nu|=n} \frac{\mathrm{H}_{\nu}(\beta ; \kappa, n)}{\mathrm{B}_{\nu}(\kappa, N)} P_{\nu, N}(y) \\
& =\frac{(\kappa+\mathbf{1})_{\beta}}{\left(\lambda_{\kappa}\right)_{n}} \sum_{|\alpha|=N} \frac{N !}{\alpha !} \sum_{|\nu|=n} \frac{\mathrm{H}_{\nu}(\beta ; \kappa, n)}{\mathrm{B}_{\nu}(\kappa, N)} \mathrm{H}_{\nu}(\alpha ; \kappa, N) y^{\alpha} \\
& =\frac{(\kappa+\mathbf{1})_{\beta}}{\left(\lambda_{\kappa}\right)_{n}} \sum_{|\alpha|=N} \frac{N !}{\alpha !} \frac{(-1)^{n}\left(\lambda_{\kappa}\right)_{n}}{(-N)_{n}(\kappa+\mathbf{1})_{\beta}} \mathrm{Q}_{\beta}(\alpha ; \kappa, N) y^{\alpha},
\end{aligned}
$$

which simplifies to the (3.3).

The generating function relation (3.3) can be used to derive an explicit expansion of $\mathrm{Q}_{\beta}(\cdot ; \kappa, N)$. The following simple lemma is useful.

Lemma 3.5. For $y \in \mathbb{Z}_{N}^{d+1}$ and $\alpha \in \mathbb{N}_{0}^{d+1}$ with $|\alpha| \leq N$,

$$
\frac{1}{(-N)_{|\alpha|}} \sum_{|\gamma|=N} \frac{N !}{\gamma !}(-\gamma)_{\alpha} y^{\gamma}=|y|^{N-|\alpha|} y^{\alpha}
$$

Proof. Using the multinomial identity we obtain that

$$
\begin{aligned}
\sum_{|\gamma|=N} \frac{N !}{\gamma !}(-\gamma)_{\alpha} y^{\gamma} & =(-1)^{|\alpha|} \sum_{|\gamma|=N} \frac{N !}{(\gamma-\alpha) !} y^{\gamma} \\
& =(-1)^{|\alpha|} \sum_{|\gamma|=N-|\alpha|} \frac{N !}{\gamma !} y^{\gamma+\alpha}=\frac{(-1)^{|\gamma|} N !}{(N-|\alpha|) !}|y|^{N-|\alpha|} y^{\alpha}
\end{aligned}
$$

which proves the stated identity.

Theorem 3.6. For $\alpha \in \mathbb{N}_{0}^{d+1}$,

$$
\mathrm{Q}_{\alpha}(x ; \kappa, N)=\frac{(-N)_{|\alpha|}(\kappa+\mathbf{1})_{\alpha}}{(|\kappa|+d+|\alpha|)_{|\alpha|}} \sum_{\gamma \leq \alpha} \frac{(-\alpha)_{\gamma}(|\kappa|+d+|\alpha|)_{|\gamma|}(-1)^{|\gamma|}}{\gamma !(\kappa+\mathbf{1})_{\gamma}(-N)_{|\gamma|}} \mathrm{m}_{\alpha}(x) \text {. }
$$

Proof. By the expansion of $R_{\alpha}^{\kappa}$ and (3.4),

$$
\begin{aligned}
|y|^{N} R_{\alpha}\left(y^{\prime}\right) & =\frac{(-1)^{|\alpha|}(\kappa+\mathbf{1})_{\alpha}}{(|\kappa|+d+|\alpha|)_{|\alpha|}} \sum_{\gamma \leq \alpha} \frac{(-\alpha)_{\gamma}(|\kappa|+d+|\alpha|)_{|\gamma|}}{\gamma !(\kappa+\mathbf{1})_{\gamma}}|y|^{N-|\gamma|} y^{\gamma} \\
& =\sum_{|\beta|=N} \frac{N !}{\beta !}\left[\frac{(-1)^{|\alpha|}(\kappa+\mathbf{1})_{\alpha}}{(|\kappa|+d+|\alpha|)_{|\alpha|}} \sum_{\gamma \leq \alpha} \frac{(-\alpha)_{\gamma}(|\kappa|+d+|\alpha|)_{|\gamma|}(-\beta)_{\gamma}}{\gamma !(\kappa+\mathbf{1})_{\gamma}(-N)_{|\gamma|}}\right] X^{\beta} .
\end{aligned}
$$

Comparing with (3.3) proves (3.5). 
Proposition 3.7. For $x \in \mathbb{Z}_{N}^{d+1}$ and $\alpha \in \mathbb{N}_{0}^{d+1}$ with $|\alpha| \leq N$,

$$
\mathrm{m}_{\alpha}(x)=(-1)^{|\alpha|} \sum_{\beta \leq \alpha} \frac{(-\alpha)_{\beta}(-N)_{|\alpha|}(\kappa+\mathbf{1})_{\alpha}\left(\lambda_{\kappa}\right)_{2|\beta|}}{\beta !(-N)_{|\beta|}(\kappa+\mathbf{1})_{\beta}\left(\lambda_{\kappa}\right)_{|\alpha|+|\beta|}} \mathrm{Q}_{\beta}(x ; \kappa, N),
$$

where $\lambda_{\kappa}=|\kappa|+d+1$ as before.

Proof. Using the expansion of $Y^{\alpha}$ in terms of $R_{\beta}^{\kappa}$ and the generating function relation (3.3), we obtain, for $x \in \mathbb{R}^{d}$ and $X=(x, 1-|x|)$, that

$$
\begin{aligned}
X^{\alpha} & =(\kappa+\mathbf{1})_{\alpha} \sum_{\beta \leq \alpha} \sum_{\beta \leq \alpha} \frac{(-1)^{|\beta|}(-\alpha)_{\beta}\left(\lambda_{\kappa}\right)_{2|\beta|}}{\beta !(\kappa+\mathbf{1})_{\beta}\left(\lambda_{\kappa}\right)_{|\alpha|+|\beta|}} R_{\beta}^{\kappa}(y) \\
& =\sum_{|\gamma|=N} \frac{N !}{\gamma !}\left[\sum_{\beta \leq \alpha} \frac{(-\alpha)_{\beta}(\kappa+\mathbf{1})_{\alpha}\left(\lambda_{\kappa}\right)_{2|\beta|}}{\beta !(-N)_{|\beta|}(\kappa+\mathbf{1})_{\beta}\left(\lambda_{\kappa}\right)_{|\alpha|+|\beta|}} \mathrm{Q}_{\beta}(x ; \kappa, N)\right] X^{\gamma} .
\end{aligned}
$$

Setting $X=y /|y|$ with $y \in \mathbb{R}^{d+1}$, we obtain an expansion of $|y|^{N-|\alpha|} y^{\alpha}$ in terms of $y^{\gamma}$, which implies, when comparing with (3.4), the identity (3.6).

Definition 3.8. For $n=0,1, \ldots, N$, let $\operatorname{proj}_{\mathcal{V}_{n}^{d}\left(\mathrm{H}_{\kappa, N}\right)}$ denote the orthogonal projection from $\Pi_{N}^{d}$ onto $\mathcal{V}_{n}^{d}\left(\mathrm{H}_{\kappa, N}\right)$. For $\alpha \in \mathbb{N}_{0}^{d+1}$ with $n \leq|\alpha| \leq N$, define

$$
\mathrm{Q}_{\alpha, n}(x ; \kappa, N):=\frac{1}{(\kappa+\mathbf{1})_{\alpha}} \operatorname{proj}_{\mathcal{V}_{n}^{d}\left(\mathrm{H}_{\kappa, N}\right)} \mathrm{m}_{\alpha}(x), \quad x \in \mathbb{Z}_{N}^{d+1} .
$$

If $|\alpha|=n$, then $\mathrm{Q}_{\alpha, n}(\cdot ; \kappa, N)=\mathrm{Q}_{\alpha}(\cdot ; \kappa, N) /(\kappa+\mathbf{1})_{\alpha}$. For $|\alpha| \geq n$, it follows from (3.6) that $\mathrm{Q}_{\alpha, n}(x ; \kappa, N)$ has the following expansion:

Proposition 3.9. For $\alpha \in \mathbb{N}_{0}^{d+1}$ with $|a| \leq N$ and $n=0,1, \ldots, N$,

$$
\mathrm{Q}_{\alpha, n}(x ; \kappa, N)=\frac{(-1)^{|\alpha|}(-N)_{|\alpha|}\left(\lambda_{\kappa}\right)_{2 n}}{(-N)_{n}\left(\lambda_{\kappa}\right)_{|\alpha|+n}} \sum_{|\beta|=n} \frac{(-\alpha)_{\beta}}{\beta !(\kappa+\mathbf{1})_{\beta}} \mathrm{Q}_{\beta}(x ; \kappa, N) .
$$

Evidently, for fixed $n$ and $m \geq n$, the cardinality of the set $\left\{\mathrm{Q}_{\alpha, n}(\cdot ; \kappa, N):|\alpha|=m\right\}$ is much larger than the dimension of the $\mathcal{V}_{n}^{d}\left(\mathrm{H}_{\kappa, N}\right)$. Hence, there are ample redundancy in the set. We will need explicit formulas for $\mathrm{Q}_{\alpha, n}(x ; \kappa, N)$ for which the following function defined in [19] is useful.

Definition 3.10. Let $\kappa \in \mathbb{R}^{d+1}$ with $\kappa_{i}>-1$. For $x, y \in \mathbb{Z}_{N}^{d+1}$, and $n=0,1, \ldots$, define $\mathcal{E}_{0}(x, y ; \kappa)=1$ and

$$
\mathcal{E}_{n}(x, y ; \kappa):=\sum_{|\gamma|=n} \frac{(-x)_{\gamma}(-y)_{\gamma}}{\gamma !(\kappa+\mathbf{1})_{\gamma}}, \quad n=1,2, \ldots, N .
$$

Proposition 3.11. For $m \geq n, \alpha \in \mathbb{Z}_{m}^{d+1}$ and $x \in \mathbb{Z}_{N}^{d+1}$,

$$
\begin{aligned}
\mathrm{Q}_{\alpha, n}(x ; \kappa, N)= & \frac{(-N)_{m}(-m)_{n}(|\kappa|+d+1)_{n}(|\kappa|+d+2 n)}{n !(|\kappa|+d+1)_{m+n}(|\kappa|+d+n)} \\
& \times \sum_{k=1}^{n} \frac{(-n)_{k}(|\kappa|+d+n)_{k}}{(-m)_{k}(-N)_{k}} \mathcal{E}_{k}(\alpha, x ; \kappa) .
\end{aligned}
$$


Proof. Using $\lambda_{\kappa}=|\kappa|+d+1$, it follows from (3.7) and (3.5) that

$$
\mathrm{Q}_{\alpha, n}(x ; \kappa, N)=\frac{(-1)^{m}(-N)_{m}\left(\lambda_{\kappa}\right)_{2 n}}{\left(\lambda_{\kappa}\right)_{m+n}(|\kappa|+d+n)_{n}} \sum_{|\beta|=n} \frac{(-\alpha)_{\beta}}{\beta !} \sum_{\gamma \leq \beta} \frac{(-\beta)_{\gamma}(|\kappa|+d+n)_{|\gamma|} \mathrm{m}_{\gamma}(x)}{\gamma !(\kappa+\mathbf{1})_{\gamma}(-N)_{|\gamma|}} .
$$

Since $(-\gamma)_{\beta}=0$ whenever $\gamma>\beta$, we can write the sum over $\gamma \leq \beta$ as $\gamma \in \mathbb{N}_{0}^{d+1}$, so that we can consider the sum over $|\beta|=n$ first. By the multinomial identity,

$$
\begin{aligned}
\sum_{|\beta|=n} \frac{(-\alpha)_{\beta}(-\beta)_{\gamma}}{\beta !} & =\sum_{|\beta|=n} \frac{(-\alpha)_{\beta}(-1)^{|\gamma|}}{(\beta-\gamma) !}=(-1)^{|\gamma|} \sum_{|\beta|=n-|\gamma|} \frac{(-\alpha)_{\beta+\gamma}}{\beta !} \\
& =(-1)^{|\gamma|}(-\alpha)_{\gamma} \sum_{|\beta|=n-|\gamma|} \frac{(-\alpha+\gamma)_{\beta}}{\beta !}=(-1)^{|\gamma|}(-\alpha)_{\gamma} \frac{(-|\alpha|+|\gamma|)_{n-|\gamma|}}{(n-|\gamma|) !} \\
& =\frac{(-\alpha)_{\gamma}(-|\alpha|)_{n}(-n)_{|\gamma|}}{(-|\alpha|)_{|\gamma|} n !} .
\end{aligned}
$$

Consequently, we obtain

$$
\mathrm{Q}_{\alpha, n}(x ; \kappa, N)=\frac{(-1)^{m}(-N)_{m}\left(\lambda_{\kappa}\right)_{2 n}(-m)_{n}}{\left(\lambda_{\kappa}\right)_{m+n}(|\kappa|+d+n)_{n} n !} \sum_{\gamma} \frac{(-n)_{|\gamma|}(|\kappa|+d+n)_{|\gamma|}(-\alpha)_{\gamma} \mathrm{m}_{\gamma}(x)}{(-m)_{|\gamma|}(-N)_{|\gamma|} \gamma !(\kappa+\mathbf{1})_{\gamma}} .
$$

Simplifying the constant in front and writing the summation over $\gamma$ as $\sum_{k=0}^{n} \sum_{|\gamma|=k}$, we then obtain (3.8).

Proposition 3.12. For $m \geq n, \alpha \in \mathbb{Z}_{m}^{d+1}$ and $\nu \in \mathbb{N}_{0}^{d}$ with $|\nu|=n$,

$$
\mathrm{Q}_{\alpha, n}(x ; \kappa, N)=\frac{(-1)^{m}(-m)_{n}(-N)_{m}\left(\lambda_{\kappa}\right)_{N+n}}{(-N)_{n}\left(\lambda_{\kappa}\right)_{N}\left(\lambda_{\kappa}\right)_{m+n}} \sum_{|\nu|=n} \frac{\mathrm{H}_{\nu}(\alpha ; \kappa, m)}{\mathrm{B}_{\nu}(\kappa, N)} \mathrm{H}_{\nu}(x ; \kappa, N) .
$$

Conversely,

$$
\mathrm{H}_{\nu}(x ; \kappa, N)=\frac{(-N)_{m}}{m !} \sum_{|\alpha|=m} \frac{(\kappa+\mathbf{1})_{\alpha}}{\alpha !} \mathrm{H}_{\nu}(\alpha ; \kappa, m) \mathrm{Q}_{\alpha, n}(x ; \kappa, N) .
$$

Proof. From (3.7) and (3.2), it follows that

$$
\mathrm{Q}_{\alpha, n}(x ; \kappa, N)=\frac{(-1)^{m+n}(-N)_{m}\left(\lambda_{\kappa}\right)_{2 n}}{\left(\lambda_{\kappa}\right)_{m+n}\left(\lambda_{\kappa}\right)_{n}} \sum_{|\beta|=n} \frac{(-\alpha)_{\beta}}{\beta !} \sum_{|\nu|=n} \frac{\mathrm{H}_{\nu}(\beta ; \kappa, n)}{\mathrm{B}_{\nu}(\kappa, N)} \mathrm{H}_{\nu}(x ; \kappa, N) .
$$

Exchanging the order of summations and applying (2.7), we obtain (3.9) after simplifying the constants. Conversely, we use the orthogonality of $\mathrm{H}_{\nu}(\cdot ; \kappa, m)$ and deduce from (3.9) that

$$
\begin{aligned}
\sum_{|\alpha|=m} & \frac{(\kappa+\mathbf{1})_{\alpha}}{\alpha !} \mathrm{H}_{\nu}(\alpha ; \kappa, m) \mathrm{Q}_{\alpha, n}(x ; \kappa, N) \\
\quad= & \frac{(-m)_{n}(-N)_{m}\left(\lambda_{\kappa}\right)_{N+n}}{(-N)_{n}\left(\lambda_{\kappa}\right)_{N}\left(\lambda_{\kappa}\right)_{m+n}} \frac{\left(\lambda_{k}\right)_{m}}{m !} \frac{\mathrm{B}_{\nu}(\kappa, m)}{\mathrm{B}_{\nu}(\kappa, N)} \mathrm{H}_{\nu}(x ; \kappa, N) .
\end{aligned}
$$

Simplifying the constant by (2.6) proves (3.10). 
In particular, (3.10) shows that the set $\left\{\mathrm{Q}_{\alpha, n}(\cdot ; \kappa, N):|\alpha|=m\right\}$, which is a subset of the space $\mathcal{V}_{n}^{d}\left(\mathrm{H}_{\kappa, N}\right)$, spans the space.

Let $\mathrm{P}_{n}\left(\mathrm{H}_{\kappa, N} ; \cdot, \cdot\right)$ denote the reproducing kernel of $\mathcal{V}_{n}^{d}\left(\mathrm{H}_{\kappa, N}\right)$, which is characterized by the requirement that it is an element of $\mathcal{V}_{n}^{d}\left(\mathrm{H}_{\kappa, N}\right)$ in either its variable and

$$
\left\langle\mathrm{P}_{n}\left(\mathrm{H}_{\kappa, N} ; x, \cdot\right), \mathrm{P}\right\rangle_{\mathrm{H}_{\kappa, N}}=\mathrm{P}(x), \quad \forall \mathrm{P} \in \mathcal{V}_{n}^{d}\left(\mathrm{H}_{\kappa, N}\right) .
$$

In terms of the mutually orthogonal basis $\left\{\mathrm{H}_{\nu}(\cdot ; \kappa, N):|\nu|=n\right\}$, the reproducing kernel can be written as

$$
\mathrm{P}_{n}\left(\mathrm{H}_{\kappa, N} ; x, y\right)=\sum_{|\nu|=n} \frac{\mathrm{H}_{\nu}(x ; \kappa, N) \mathrm{H}_{\nu}(y ; \kappa, N)}{\mathrm{B}_{\nu}(\kappa, N)} .
$$

Our next result shows that this kernel can be expanded in $\mathrm{Q}_{\alpha, n}(\cdot ; \kappa, N)$.

Theorem 3.13. For $m \geq n, \alpha \in \mathbb{Z}_{m}^{d+1}$ and $x, y \in \mathbb{Z}_{N}^{d+1}$,

$$
\mathrm{P}_{n}\left(\mathrm{H}_{\kappa, N} ; x, y\right)=\mathrm{D}_{n}(m, N) \sum_{|\alpha|=m} \frac{(\kappa+\mathbf{1})_{\alpha}}{\alpha !} \mathrm{Q}_{\alpha, n}(x ; \kappa, N) \mathrm{Q}_{\alpha, n}(y ; \kappa, N) \text {, }
$$

where

$$
\mathrm{D}_{n}(m, N):=\frac{(-N)_{n} m !(|\kappa|+d+1)_{N}(|\kappa|+d+1)_{m+n}}{(-m)_{n}\left[(-N)_{m}\right]^{2}(|\kappa|+d+1)_{N+n}} .
$$

Proof. Let $C_{n}(m, N)$ denote the constant in from of the sum in (3.9). Then

$$
\begin{aligned}
& \sum_{|\alpha|=m} \frac{(\kappa+\mathbf{1})_{\alpha}}{\alpha !} \mathrm{Q}_{\alpha, n}(x ; \kappa, N) \mathrm{Q}_{\alpha, n}(y ; \kappa, N) \\
& \quad=\left[C_{n}(m, N)\right]^{2} \sum_{|\nu|=n} \sum_{|\mu|=n} \frac{\mathrm{H}_{\nu}(x ; \kappa, N) \mathrm{H}_{\mu}(y ; \kappa, N)}{\mathrm{B}_{\nu}(\kappa, N) \mathrm{B}_{\mu}(\kappa, N)} \sum_{|\alpha|=m} \frac{(\kappa+\mathbf{1})_{\alpha}}{\alpha !} \mathrm{H}_{\mu}(\alpha ; \kappa, m) \mathrm{H}_{\nu}(\alpha ; \kappa, m) \\
& \quad=C_{n}(m, N)^{2} \sum_{|\nu|=n} \frac{\mathrm{H}_{\nu}(x ; \kappa, N) \mathrm{H}_{\nu}(y ; \kappa, N)}{\mathrm{B}_{\nu}(\kappa, N)} \frac{\left(\lambda_{k}\right)_{m} \mathrm{~B}_{\nu}(\kappa, m)}{m ! \mathrm{B}_{\nu}(\kappa, N)} \\
& \quad=C_{n}(m, N)^{2} \frac{\left(\lambda_{k}\right)_{m}\left(\lambda_{\kappa}\right)_{m+n}\left(\lambda_{\kappa}\right)_{N}(-N)_{n}}{m !\left(\lambda_{\kappa}\right)_{N+n}\left(\lambda_{\kappa}\right)_{m}(-m)_{n}} \mathrm{P}_{n}\left(\mathrm{H}_{\kappa, N} ; x, y\right),
\end{aligned}
$$

where we have used (2.6). This proves (3.12) after simplifying the constants.

While (3.11) follows directly from the definition, (3.12) is by no means trivial since the set $\left\{\mathrm{Q}_{\alpha, n}(\cdot ; \kappa, N): \alpha \in \mathbb{Z}_{N}^{d+1}\right\}$ is linearly dependent and is heavily redundant. In fact, the identity (3.12) is the key result for our discussion on the tight frames in Section 5.

\section{Monic Krawtchouk polynomials of several variables}

For each $\alpha \in \mathbb{Z}_{N}^{d+1}$, we define the monic Krawtchouk polynomial as the orthogonal polynomial that has $\mathrm{m}_{\alpha}$ as its leading term.

Definition 4.1. For $\alpha \in \mathbb{N}_{0}^{d+1}$ and $|\alpha| \leq N$, the monic Krawtchouk polynomial $\mathrm{L}_{\alpha}(\cdot ; \kappa, N) \in$ $\mathcal{V}_{|\alpha|}^{d}\left(\mathrm{~K}_{\rho, N}\right)$ is defined uniquely by

$$
\mathrm{K}_{\alpha}(x ; \rho, N):=\mathrm{m}_{\alpha}(x)+q_{\alpha}(x), \quad q \in \Pi_{|\alpha|-1}^{d}, \quad x \in \mathbb{Z}_{N}^{d+1} .
$$


The properties of such polynomials can be derived from the monic Hahn polynomials $\mathrm{Q}_{\nu}(\cdot ; \kappa, N)$. Indeed, it is easy to see that

$$
\lim _{t \rightarrow \infty} t^{-N} \mathrm{H}_{\nu, t \boldsymbol{\rho}}(x)=\mathrm{K}_{\rho}(x), \quad x \in \mathbb{Z}_{N}^{d+1}, \quad \text { and } \quad \lim _{t \rightarrow \infty} \mathrm{B}_{\nu}(t \boldsymbol{\rho}, N)=\mathrm{C}_{\nu}(\rho, N) .
$$

From the first identity follows readily that the inner product $\langle f, g\rangle_{\mathrm{H}_{\kappa, N}}$ becomes $\langle f, \gamma\rangle_{\mathrm{K}_{\rho, N}}$ if we set $\kappa=t \rho$ and let $t \rightarrow \infty$. Consequently, we conclude that

Proposition 4.2. For $\rho \in(0,1)^{d}$ with $|\rho|<1, \nu \in \mathbb{N}_{0}^{d}$ and $x \in \mathbb{Z}_{N}^{d+1}$,

$$
\lim _{t \rightarrow \infty} \mathrm{Q}_{\nu}(x ; t \boldsymbol{\rho}, N)=\mathrm{L}_{\nu}(x ; \rho, N) .
$$

Together with the limit relation (2.12), we deduce following relations from Proposition 3.3.

Proposition 4.3. For $x \in \mathbb{Z}_{N}^{d+1}, \alpha \in \mathbb{Z}_{n}^{d+1}$ and $\nu \in \mathbb{N}_{0}^{d}$ with $|\nu|=n$,

$$
\mathrm{K}_{\nu}(x ; \rho, N)=\frac{(-1)^{n} n !}{(-N)_{n}} \sum_{|\alpha|=n} \frac{\mathrm{K}_{\nu}(\alpha ; \rho, n)}{\alpha !} \mathrm{L}_{\alpha}(x ; \rho, N),
$$

and, conversely,

$$
\mathrm{L}_{\alpha}(x ; \rho, N)=(-1)^{n}(-N)_{n} \rho^{\alpha} \sum_{|\nu|=n} \frac{\mathrm{K}_{\nu}(\alpha ; \rho, n)}{\mathrm{C}_{\nu}(\rho, n)} \mathrm{K}_{\nu}(x ; \rho, N) .
$$

Furthermore, from Theorem 3.6 and Proposition 3.7, we deduce the expansion of monic Krawtchouk polynomials and its converse.

Theorem 4.4. For $\alpha \in \mathbb{N}_{0}^{d+1}$,

$$
\mathrm{L}_{\alpha}(x ; \rho, N)=(-N)_{|\alpha|} \boldsymbol{\rho}^{\alpha} \sum_{\gamma \leq \alpha} \frac{(-\alpha)_{\gamma}(-1)^{|\gamma|}}{\gamma !(-N)_{|\gamma|} \boldsymbol{\rho}^{\gamma}} \mathrm{m}_{\alpha}(x) .
$$

Conversely,

$$
\mathrm{m}_{\alpha}(x)=(-1)^{|\alpha|}(-N)_{|\alpha|} \rho^{\alpha} \sum_{\beta \leq \alpha} \frac{(-\alpha)_{\beta}}{\beta !(-N)_{|\beta|} \rho^{\beta}} \mathrm{L}_{\beta}(x ; \kappa, N) .
$$

We now define analogues of $\mathrm{Q}_{\alpha, n}(x ; \kappa, N)$ polynomials.

Definition 4.5. For $n=0,1, \ldots, N$, let $\operatorname{proj}_{\mathcal{V}_{n}^{d}\left(\mathrm{~K}_{\rho, N}\right)}$ denote the orthogonal projection from $\Pi_{N}^{d}$ onto $\mathcal{V}_{n}^{d}\left(\mathrm{~K}_{\rho, N}\right)$. For $\alpha \in \mathbb{N}_{0}^{d+1}$ with $n \leq|\alpha| \leq N$, define

$$
\mathrm{L}_{\alpha, n}(x ; \kappa, N):=\frac{1}{(\kappa+\mathbf{1})_{\alpha}} \operatorname{proj}_{\mathcal{V}_{n}^{d}\left(\mathrm{~K}_{\kappa, N}\right)} \mathrm{m}_{\alpha}(x), \quad x \in \mathbb{Z}_{N}^{d+1} .
$$

If $|\alpha|=n$, then $\mathrm{L}_{\alpha, n}(\cdot ; \rho, N)=\mathrm{L}_{\alpha}(\cdot ; \rho, N) / \boldsymbol{\rho}^{\alpha}$. It follows that

$$
\lim _{t \rightarrow \infty} t^{|\alpha|} \mathrm{Q}_{\alpha, n}(x ; \kappa, N)=\mathrm{L}_{\alpha, n}(\cdot ; \rho, N) .
$$

Consequently, from Proposition 3.9, we deduce the following relation.

Proposition 4.6. For $\alpha \in \mathbb{N}_{0}^{d+1}$ with $|a| \leq N$ and $n=0,1, \ldots, N$,

$$
\mathrm{L}_{\alpha, n}(x ; \rho, N)=\frac{(-1)^{|\alpha|}(-N)_{|\alpha|}}{(-N)_{n}} \sum_{|\beta|=n} \frac{(-\alpha)_{\beta}}{\beta ! \rho^{\beta}} \mathrm{L}_{\beta}(x ; \kappa, N) .
$$


Furthermore, we derive from Proposition 3.12 the expansion of $\mathrm{L}_{\nu}(\cdot ; \rho, N)$ in terms of mutually orthogonal $\mathrm{K}_{\nu}(\cdot ; \rho, N)$ and its converse.

Proposition 4.7. For $m \geq n, \alpha \in \mathbb{Z}_{m}^{d+1}$ and $\nu \in \mathbb{N}_{0}^{d}$ with $|\nu|=n$,

$$
\mathrm{L}_{\alpha, n}(x ; \rho, N)=\frac{(-m)_{n}(-N)_{m}}{(-N)_{n}} \sum_{|\nu|=n} \frac{\mathrm{K}_{\nu}(\alpha ; \rho, m)}{\mathrm{C}_{\nu}(\rho, N)} \mathrm{K}_{\nu}(x ; \rho, N) .
$$

Conversely,

$$
\mathrm{K}_{\nu}(x ; \rho, N)=\frac{(-N)_{m}}{m !} \sum_{|\alpha|=m} \frac{\rho^{\alpha}}{\alpha !} \mathrm{K}_{\nu}(\alpha ; \rho, m) \mathrm{L}_{\alpha, n}(x ; \rho, N) .
$$

The analogue of the function $\mathcal{E}_{n}(x, y ; \kappa)$ is defined as follows:

Definition 4.8. Let $\rho \in(0,1)^{d}$ with $|\rho|<1$. For $x, y \in \mathbb{Z}_{N}^{d+1}$, and $n=0,1, \ldots$, define $\mathcal{F}_{0}(x, y ; \rho)=1$ and

$$
\mathcal{F}_{n}(x, y ; \rho):=\sum_{|\gamma|=n} \frac{(-x)_{\gamma}(-y)_{\gamma}}{\gamma ! \boldsymbol{\rho}^{\gamma}}, \quad n=1,2, \ldots, N .
$$

This function is defined in [19]. It is easy to see that

$$
\lim _{t \rightarrow \infty} t^{k} \mathcal{E}_{k}(x, y ; t \boldsymbol{\rho}, N)=\mathcal{F}_{k}(x, y ; \rho, N), \quad x, y \in \mathbb{Z}_{N}^{d+1} .
$$

Consequently, we can deduce from Proposition 3.11 an expansion of $\mathrm{L}(\nu ; \rho, N)$.

Proposition 4.9. For $m \geq n, \alpha \in \mathbb{Z}_{m}^{d+1}$ and $x \in \mathbb{Z}_{N}^{d+1}$,

$$
\mathrm{L}_{\alpha, n}(x ; \rho, N)=\frac{(-N)_{m}(-m)_{n}}{n !} \sum_{k=1}^{n} \frac{(-n)_{k}}{(-m)_{k}(-N)_{k}} \mathcal{F}_{k}(\alpha, x ; \rho) .
$$

Finally, let $\mathrm{P}_{n}\left(\mathrm{~K}_{\rho, N} ; \cdot, \cdot\right)$ denote the reproducing kernel of $\mathcal{V}_{n}^{d}\left(\mathrm{~K}_{\rho, N}\right)$. In terms of the mutually orthogonal basis $\mathrm{K}_{\nu}(\cdot ; \rho, N)$, we can write $\mathrm{P}_{n}\left(\mathrm{~K}_{\rho, N} ; \cdot, \cdot\right)$ as

$$
\mathrm{P}_{n}\left(K_{\rho, N} ; x, y\right)=\sum_{|\nu|=n} \frac{\mathrm{K}_{\nu}(x ; \rho, N) \mathrm{K}_{\nu}(y ; \rho, N)}{\mathrm{C}(\rho, N)} .
$$

It can also be expressed in $\mathrm{L}_{\alpha, n}(\cdot ; \rho, N)$ as the following analogue of Theorem 3.13 shows.

Theorem 4.10. For $m \geq n, \alpha \in \mathbb{Z}_{m}^{d+1}$ and $x, y \in \mathbb{Z}_{N}^{d+1}$,

$$
\mathrm{P}_{n}\left(\mathrm{~K}_{\rho, N} ; x, y\right)=\frac{(-N)_{n} m !}{(-m)_{n}\left[(-N)_{m}\right]^{2}} \sum_{|\alpha|=m} \frac{\rho^{\alpha}}{\alpha !} \mathrm{L}_{\alpha, n}(x ; \rho, N) \mathrm{L}_{\alpha, n}(y ; \rho, N) \text {. }
$$

\section{$5 \quad$ Finite Tight frames}

For $n \leq m \leq N$, let us consider the set

$$
\Xi_{m, n}(\kappa, N):=\left\{\mathrm{Q}_{\alpha, n}(\cdot ; \kappa, N): \alpha \in \mathbb{Z}_{m}^{d+1}\right\} .
$$

Clearly $\Xi_{m, n}(\kappa, N)$ is a subset of $\mathcal{V}_{n}^{d}\left(\mathrm{H}_{\kappa, N}\right)$. Moreover, by $(3.10)$, span $\Xi_{m, n}(\kappa, N)=\mathcal{V}_{n}^{d}\left(\mathrm{H}_{\kappa, N}\right)$. The cardinality of $\Xi_{m, n}(\kappa, N)$ is much large than the dimension of $\mathcal{V}_{n}^{d}\left(\mathrm{H}_{\kappa, N}\right)$. In fact,

$$
\frac{\# \Xi_{m, n}(\kappa, N)}{\operatorname{dim} \mathcal{V}_{n}^{d}\left(\mathrm{H}_{\kappa, N}\right)}=\frac{\left(\begin{array}{c}
m+d \\
m
\end{array}\right)}{\left(\begin{array}{c}
n+d-1 \\
n
\end{array}\right)}=\frac{m}{d}\left(\frac{m}{n}\right)^{d-1}\left(1+\mathcal{O}\left(m^{-1}\right)\right)
$$

The following theorem shows that $\Xi_{m, n}(\kappa, N)$ is a tight frame for $\mathcal{V}_{n}^{d}\left(\mathrm{H}_{\kappa, N}\right)$. 
Theorem 5.1. Let $m \geq n$. Then for all

$$
f(x)=\sum_{|\alpha|=m} \frac{(\kappa+\mathbf{1})_{\alpha}}{\alpha !}\left\langle f, \mathrm{Q}_{\alpha, n}(\cdot ; \kappa, N)\right\rangle_{\mathrm{H}_{\kappa, N}} \mathrm{Q}_{x, n}(\alpha ; \kappa, N) .
$$

Furthermore, $\left\{\mathrm{Q}_{\alpha, n}(\cdot ; \kappa, N):|\alpha|=m\right\}$ is a tight frame of $\mathcal{V}_{n}^{d}\left(\mathrm{H}_{n, N}\right)$,

$$
\langle f, f\rangle_{\mathrm{H}_{\kappa, N}}=\mathrm{D}_{n}(m, N) \sum_{|\alpha|=m} \frac{(\kappa+\mathbf{1})_{\alpha}}{\alpha !}\left\langle f, \mathrm{Q}_{\alpha, n}(\cdot ; \kappa, N)\right\rangle_{\mathrm{H}_{\kappa, N}}^{2} .
$$

Proof. If $f \in \mathcal{V}_{n}^{d}\left(\mathrm{H}_{n, N}\right)$, then $f(x)=\left\langle f, \mathrm{P}_{n}\left(\mathrm{H}_{\kappa, N} ; \cdot, x\right)\right\rangle_{\mathrm{H}_{\kappa, N}}$, so that the first identity follows immediately from (3.12). Taking the inner product of the first identity with $f$ gives the second identity.

A finite tight frame is equivalent to a tight frame in an Euclidean space. From Theorem 5.1 and connecting coefficients of our Hahn polynomials, we can derive Euclidean tight frames that can be given explicitly. Recall that $\mathrm{r}(d, n)=\left(\begin{array}{c}n+d-1 \\ n\end{array}\right)$. Fixing a linear order in the set $\left\{\nu \in \mathbb{N}_{0}^{d}\right.$ : $|\nu|=n\}$, say the lexicographical order, we denote $x \in \mathbb{R}^{r(d, n)}$ as $x=\left(x_{\nu}:|\nu|=n, \nu \in \mathbb{N}_{0}^{d}\right)$. The usual inner product in $\mathbb{R}^{r(d, n)}$ then takes the form

$$
(x, y):=\sum_{|\nu|=n} x_{\nu} y_{\nu}, \quad x, y \in \mathbb{R}^{r(d, n)}
$$

Theorem 5.2. Let $m, n=1,2, \ldots$ with $m \geq n$. For $\alpha \in \mathbb{Z}_{m}^{d+1}$, define vectors

$$
\mathrm{h}_{\alpha, n}=\sqrt{\frac{m !(\kappa+\mathbf{1})_{\alpha}}{(|\kappa|+d+1)_{m} \alpha !}}\left(\frac{\mathrm{H}_{\nu}(\alpha, \kappa, m)}{\sqrt{\mathrm{B}_{\nu}(\kappa, m)}}:|\nu|=n\right) \in \mathbb{R}^{\mathrm{r}(d, n)} .
$$

Then the set $\mathrm{H}(d, n, m, \kappa):=\left\{\mathrm{h}_{\alpha, n}:|\alpha|=m\right\}$ is a tight frame in $\mathbb{R}^{\mathrm{r}(d, n)}$, that is,

$$
\|x\|^{2}=(x, x)=\sum_{|\alpha|=m}\left(x, \mathrm{~h}_{\alpha, n}\right)^{2}, \quad \forall x \in \mathbb{R}^{r(d, n)},
$$

and the frame has $\# \mathbf{H}(d, n, m, \kappa)=\left(\begin{array}{c}m+d \\ d\end{array}\right)$ elements.

Proof. For $y \in \mathbb{R}^{r(d, n)}$ and $x \in \mathbb{Z}_{N}^{d+1}$, define

$$
f_{y}(x)=\sum_{|\nu|=n} y_{\nu} \frac{\mathrm{H}_{\nu}(x ; \kappa, N)}{\sqrt{\mathrm{B}_{\nu}(\kappa, N)}} .
$$

By the orthogonality of $\mathrm{H}_{\nu}(x ; \kappa, N)$, we immediately have $\left\langle f_{y}, f_{y}\right\rangle_{\mathrm{H}_{\kappa, N}}=(y, y)$. On the other hand, by (3.9), we obtain

$$
\left\langle f_{y}, \mathrm{Q}_{\alpha, n}(\cdot ; \kappa, N)\right\rangle_{\mathrm{H}_{\kappa, N}}=C_{n}(m, N) \sum_{|\nu|=n} y_{\nu} \frac{\mathrm{H}_{\nu}(\alpha ; \kappa, m)}{\sqrt{\mathrm{B}_{\nu}(\kappa, m)}} \frac{\sqrt{\mathrm{B}_{\nu}(\kappa, m)}}{\sqrt{\mathrm{B}_{\nu}(\kappa, N)}},
$$

where $C_{n}(m, N)$ is the constant in front of the summation in (3.9). Using (2.6), it follows readily that

$$
\left\langle f_{y}, \mathrm{Q}_{\alpha, n}(\cdot ; \kappa, N)\right\rangle_{\mathrm{H}_{\kappa, N}}^{2}=C_{n}(m, N)^{2} \frac{\left(\lambda_{\kappa}\right)_{m+n}\left(\lambda_{\kappa}\right)_{N}(-N)_{n}}{\left(\lambda_{\kappa}\right)_{N+n}\left(\lambda_{\kappa}\right)_{m}(-m)_{n}} \frac{(|\kappa|+d+1)_{m} \alpha !}{m !(\kappa+\mathbf{1})_{\alpha}}\left(y, \mathrm{~h}_{\alpha, n}\right)^{2} .
$$

Substituting into (5.1) and simplifying the constant, we see that the right hand side of (5.1) becomes exactly $\sum_{|\alpha|=m}\left(y, \mathrm{~h}_{\alpha, n}\right)^{2}$. 
Recall that $\mathrm{P}_{n}\left(\mathrm{H}_{\kappa, m} ; \cdot, \cdot\right)$ denotes the reproducing kernel of $\mathcal{V}_{n}^{d}\left(\mathrm{H}_{\kappa, m}\right)$.

Corollary 5.3. Let $\mathrm{h}_{\alpha, n}$ be the vectors defined in the previous theorem. Then

$$
\left(\mathrm{h}_{\alpha, n}, \mathrm{~h}_{\beta, n}\right)=\mathrm{P}_{n}\left(\mathrm{H}_{\kappa, m} ; \alpha, \beta\right), \quad \alpha, \beta \in \mathbb{Z}_{m}^{d+1} \text {. }
$$

In particular, in terms of the unit vectors $\widetilde{\mathrm{h}}_{\alpha, n}=\mathrm{h}_{\alpha, n} /\left\|\mathrm{h}_{\alpha, n}\right\|$,

$$
\|x\|^{2}=\sum_{|\alpha|=n} \mathrm{P}_{n}\left(\mathrm{H}_{\kappa, m} ; \alpha, \alpha\right)\left(x, \widetilde{\mathrm{h}}_{\alpha, n}\right)^{2}, \quad \forall x \in \mathbb{R}^{\mathrm{r}(d, m)} .
$$

Proof. The identity (5.2) follows directly from the definition of $\mathrm{h}_{\alpha, n}$ and (3.11).

The tight frames in $\mathrm{H}(d, n, m, \kappa)$ are given by explicit formulas. Using the expressions $\mathrm{H}_{\nu}(x ; \kappa, m)$ in $(2.4)$ and $\mathrm{B}_{\nu}(\kappa, m)$ in $(2.5)$, the vectors in the frame can be easily computed. In the case of $d=2, \mathrm{H}(2, n, m, \kappa)$ is a tight frame of $\mathbb{R}^{n+1}$ with $(m+2)(m+1) / 2$ elements. Below are several examples for $d=2$ and $\kappa=0$, in which the vectors in $\mathrm{H}(2, n, m, \kappa)$ are column of a matrix, which we again call $\mathrm{H}(2, n, m, \kappa)$.

Example 5.4. For $d=2$ and $\kappa=0$, set $\mathbf{H}(n, m)=\mathbf{H}(2, n, m, 0)$. Then

$$
\mathrm{H}(2,2)=\left[\begin{array}{cccccc}
\frac{1}{\sqrt{6}} & -\sqrt{\frac{2}{3}} & 0 & \frac{1}{\sqrt{6}} & 0 & 0 \\
\frac{1}{\sqrt{10}} & 0 & -\sqrt{\frac{2}{5}} & -\frac{1}{\sqrt{10}} & \sqrt{\frac{2}{5}} & 0 \\
\frac{1}{\sqrt{30}} & \frac{1}{\sqrt{30}} & -\sqrt{\frac{3}{10}} & \frac{1}{\sqrt{30}} & -\sqrt{\frac{3}{10}} & \sqrt{\frac{3}{10}}
\end{array}\right]
$$

is a tight frame in $\mathbb{R}^{3}$ with 6 elements,

$$
\mathrm{H}(2,3)=\left[\begin{array}{cccccccccc}
\sqrt{\frac{3}{14}} & -\sqrt{\frac{3}{14}} & \frac{3}{\sqrt{42}} & -\sqrt{\frac{3}{14}} & -\sqrt{\frac{2}{21}} & 0 & \sqrt{\frac{3}{14}} & \frac{3}{\sqrt{42}} & 0 & 0 \\
\frac{3}{\sqrt{70}} & \frac{1}{\sqrt{70}} & -\frac{3}{\sqrt{70}} & -\frac{1}{\sqrt{70}} & 0 & -\frac{4}{\sqrt{70}} & -\frac{3}{\sqrt{70}} & \frac{3}{\sqrt{70}} & \frac{4}{\sqrt{70}} & 0 \\
\sqrt{\frac{3}{70}} & \sqrt{\frac{3}{70}} & -\sqrt{\frac{5}{42}} & \sqrt{\frac{3}{70}} & -\sqrt{\frac{5}{42}} & -\sqrt{\frac{3}{70}} & \sqrt{\frac{3}{70}} & -\sqrt{\frac{5}{42}} & -\sqrt{\frac{3}{70}} & \sqrt{\frac{27}{70}}
\end{array}\right]
$$

is a tight frame in $\mathbb{R}^{3}$ with 10 elements, and

$$
\mathrm{H}(3,3)=\left[\begin{array}{cccccccccc}
\frac{-1}{2 \sqrt{5}} & \frac{3}{2 \sqrt{5}} & 0 & -\frac{3}{2 \sqrt{5}} & 0 & 0 & \frac{1}{2 \sqrt{5}} & 0 & 0 & 0 \\
\frac{-1}{2 \sqrt{7}} & \frac{-1}{2 \sqrt{7}} & \frac{-1}{\sqrt{7}} & \frac{1}{2 \sqrt{7}} & \frac{-2}{\sqrt{7}} & 0 & \frac{-1}{2 \sqrt{7}} & \frac{1}{\sqrt{7}} & 0 & 0 \\
\frac{-\sqrt{3}}{2 \sqrt{35}} & \frac{-1}{2 \sqrt{105}} & \sqrt{\frac{5}{21}} & \frac{1}{2 \sqrt{105}} & 0 & -\sqrt{\frac{5}{21}} & \frac{\sqrt{3}}{2 \sqrt{35}} & -\sqrt{\frac{5}{21}} & \sqrt{\frac{5}{21}} & 0 \\
\frac{-1}{2 \sqrt{35}} & \frac{-1}{2 \sqrt{35}} & \frac{2}{\sqrt{35}} & \frac{-1}{2 \sqrt{35}} & \frac{2}{\sqrt{35}} & -\frac{3}{\sqrt{35}} & \frac{-1}{2 \sqrt{35}} & \frac{2}{\sqrt{35}} & \frac{-3}{\sqrt{35}} & \frac{-2}{\sqrt{35}}
\end{array}\right]
$$

is a tight frame in $\mathbb{R}^{4}$ with 10 elements.

For fixed $d, n, m$, we obtain a family of tight frames in $\mathbb{R}^{\mathrm{r}(d, n)}$ with $d+1$ parameters $\kappa=\left(\kappa_{1}, \ldots, \kappa_{d+1}\right)$. Although the proof is established for $\kappa_{i}>-1$, the analytic continuation shows that we obtain a tight frame for $\kappa \in \mathbb{R}^{d+1}$ as long as $h_{\alpha, n}$ is well defined. A frame is called normalized tight frame if all elements of the frame have the same norm [2]. Our tight frame is not normalized in general. In the case of $d=2$ and $m=n=2$, we can choose $\kappa$ to obtain a normalized tight frame. 
Example 5.5. In the case of $d=2, m=n=2$, choosing $\kappa_{1}=\kappa_{2}=\kappa_{3}=\frac{1}{2} \sqrt{-7-\sqrt{17}}$, then the row vectors in

$$
\mathrm{H}(2,2,2, \kappa)=\left[\begin{array}{ccc}
\frac{1}{2} \sqrt{-3+\sqrt{17}} & \frac{1}{4} \sqrt{-1+\sqrt{17}} & \frac{1}{4} \sqrt{21-5 \sqrt{17}} \\
\frac{\sqrt{5-\sqrt{17}}}{\sqrt{2}} & 0 & \frac{1}{2} \sqrt{-4+\sqrt{17}} \\
0 & -\frac{1}{4} \sqrt{-3+\sqrt{17}} & -\frac{1}{4} \sqrt{-1+\sqrt{17}} \\
\frac{1}{2} \sqrt{-3+\sqrt{17}} & -\frac{1}{4} \sqrt{-1+\sqrt{17}} & \frac{1}{4} \sqrt{21-5 \sqrt{17}} \\
0 & \frac{1}{4} \sqrt{-3+\sqrt{17}} & -\frac{1}{4} \sqrt{-1+\sqrt{17}} \\
0 & 0 & \sqrt{\frac{1}{2}}
\end{array}\right]
$$

form a normalized tight frame in $\mathbb{R}^{3}$ with 6 vectors.

For the Krawtchouk polynomials, the analogue of Theorem 5.1 is as follows.

Theorem 5.6. Let $m \geq n$. Then for all

$$
f(x)=\frac{(-N)_{m}(-m)_{n}}{n !} \sum_{|\alpha|=m} \frac{\rho^{\alpha}}{\alpha !}\left\langle f, \mathrm{~K}_{\alpha, n}(\cdot ; \rho, N)\right\rangle_{\mathrm{K}_{\rho, N}} \mathrm{~K}_{x, n}(\alpha ; \rho, N) .
$$

Furthermore, $\left\{\mathrm{Q}_{\alpha, n}(\cdot ; \kappa, N):|\alpha|=m\right\}$ is a tight frame of $\mathcal{V}_{n}^{d}\left(\mathrm{H}_{n, N}\right)$,

$$
\langle f, f\rangle_{\mathrm{K}_{\rho, N}}=\frac{(-N)_{m}(-m)_{n}}{n !} \sum_{|\alpha|=m} \frac{\rho^{\alpha}}{\alpha !}\left\langle f, \mathrm{~K}_{\alpha, n}(\cdot ; \rho, N)\right\rangle_{\mathrm{K}_{\rho, N}}^{2} .
$$

From Theorem 5.6 and using the connecting relation in (4.1), we obtain the following analogue of Theorem 5.2 on tight frames in Euclidean spaces.

Theorem 5.7. Let $m, n=1,2, \ldots$ with $m \geq n$. For $\alpha \in \mathbb{Z}_{m}^{d+1}$, define vectors

$$
\mathrm{k}_{\alpha, n}=\sqrt{\frac{m ! \rho^{\alpha}}{\alpha !}}\left(\frac{\mathrm{K}_{\nu}(\alpha, \rho, m)}{\sqrt{\mathrm{C}_{\nu}(\rho, m)}}:|\nu|=n\right) \in \mathbb{R}^{\mathrm{r}(d, n)} .
$$

Then the set $\mathrm{K}(d, n, m, \rho):=\left\{\mathrm{k}_{\alpha, n}:|\alpha|=m\right\}$ is a tight frame in $\mathbb{R}^{\mathrm{r}(d, n)}$, that is,

$$
(x, x)=\sum_{|\alpha|=m}\left(x, \mathrm{k}_{\alpha, n}\right)^{2}
$$

with $\# \mathrm{~K}(d, n, m, \rho)=\left(\begin{array}{c}m+d \\ d\end{array}\right)$ elements. Furthermore, for $\alpha, \beta \in \mathbb{Z}_{N}^{d+1}$,

$$
\left(\mathrm{k}_{\alpha, n}, \mathrm{k}_{\beta, n}\right)=\mathrm{P}_{n}\left(\mathrm{~K}_{\rho, m} ; \alpha, \beta\right) .
$$

This theorem can also be deduced from Theorem 5.2 by setting $\kappa=t \rho$ and taking the limit $t \rightarrow \infty$. The limiting process shows that the tight frames $\mathrm{K}(d, n, m, \rho)$ are not included in $\mathrm{H}(d, n, m, \kappa)$. In terms of explicit formulas, the Krawtchouk polynomials are simpler than the Hahn polynomials.

The tight frames $\mathrm{K}(d, n, m, \rho)$ are given by explicit formulas. Using the expression of $\mathrm{K}_{\nu}(x ; \rho, m)$ in $(2.10)$ and $\mathrm{C}_{\nu}(\rho, m)$ in $(2.11)$, the frame elements can be computed easily. Below are several examples for $d=2$ and $\rho=\left(\frac{1}{3}, \frac{1}{3}\right)$, in which the vectors in $\mathrm{K}(2, n, m, \kappa)$ are column of a matrix, which we again call $\mathrm{K}(2, n, m, \kappa)$. 
Example 5.8. For $d=2$ and $\kappa=0$, set $\mathrm{K}(n, m)=\mathrm{K}(2, n, m, 1 / 3,1 / 3)$. Then

$$
\mathrm{K}(2,2)=\left[\begin{array}{cccccc}
\frac{1}{2} & -\frac{1}{\sqrt{2}} & 0 & \frac{1}{2} & 0 & 0 \\
\frac{1}{\sqrt{6}} & 0 & -\frac{1}{\sqrt{3}} & -\frac{1}{\sqrt{6}} & \frac{1}{\sqrt{3}} & 0 \\
\frac{1}{6} & \frac{1}{3 \sqrt{2}} & -\frac{\sqrt{2}}{3} & \frac{1}{6} & -\frac{\sqrt{2}}{3} & \frac{2}{3}
\end{array}\right]
$$

is a tight frame in $\mathbb{R}^{3}$ with 6 elements,

$$
\mathrm{K}(2,3)=\left[\begin{array}{cccccccccc}
\frac{1}{2} & -\frac{1}{2 \sqrt{3}} & \frac{1}{2 \sqrt{3}} & -\frac{1}{2 \sqrt{3}} & -\frac{1}{\sqrt{6}} & 0 & \frac{1}{\sqrt{2}} & \frac{1}{2 \sqrt{3}} & 0 & 0 \\
\frac{1}{\sqrt{6}} & \frac{1}{3 \sqrt{2}} & -\frac{1}{3 \sqrt{2}} & -\frac{1}{3 \sqrt{2}} & 0 & -\frac{\sqrt{2}}{3} & -\frac{1}{\sqrt{6}} & \frac{1}{3 \sqrt{2}} & \frac{\sqrt{2}}{3} & 0 \\
\frac{1}{6} & \frac{1}{2 \sqrt{3}} & -\frac{1}{2 \sqrt{3}} & \frac{1}{2 \sqrt{3}} & -\frac{1}{\sqrt{6}} & 0 & \frac{1}{6} & -\frac{1}{2 \sqrt{3}} & 0 & \frac{2}{3}
\end{array}\right]
$$

is a tight frame in $\mathbb{R}^{3}$ with 10 elements, and

$$
\mathrm{K}(3,3)=\left[\begin{array}{cccccccccc}
-\frac{1}{2 \sqrt{2}} & \frac{\sqrt{3}}{2 \sqrt{2}} & 0 & -\frac{\sqrt{3}}{2 \sqrt{2}} & 0 & 0 & \frac{1}{2 \sqrt{2}} & 0 & 0 & 0 \\
-\frac{1}{2 \sqrt{2}} & -\frac{1}{2 \sqrt{6}} & -\frac{1}{\sqrt{6}} & \frac{1}{2 \sqrt{6}} & -\frac{1}{\sqrt{3}} & 0 & -\frac{1}{2 \sqrt{2}} & \frac{1}{\sqrt{6}} & 0 & 0 \\
-\frac{1}{2 \sqrt{6}} & -\frac{1}{6 \sqrt{2}} & -\frac{\sqrt{2}}{3} & \frac{1}{6 \sqrt{2}} & 0 & -\frac{\sqrt{2}}{3} & \frac{1}{2 \sqrt{6}} & -\frac{\sqrt{2}}{3} & -\frac{\sqrt{2}}{3} & 0 \\
-\frac{1}{6 \sqrt{6}} & -\frac{1}{6 \sqrt{2}} & \frac{1}{3 \sqrt{2}} & -\frac{1}{6 \sqrt{2}} & \frac{1}{3} & -\frac{\sqrt{2}}{3} & -\frac{1}{6 \sqrt{6}} & \frac{1}{3 \sqrt{2}} & -\frac{\sqrt{2}}{3} & \frac{2 \sqrt{2}}{3 \sqrt{3}}
\end{array}\right]
$$

is a tight frame in $\mathbb{R}^{4}$ with 10 elements.

Using the tight frames of $\mathcal{V}_{n}^{d}\left(\mathrm{H}_{\kappa, N}\right)$ as building blocks, we can also build tight frame for the space $\Pi_{N}^{d}$ of polynomials of degree at most $N$ in $d$-variables under the inner product $\langle\cdot, \cdot\rangle_{\mathrm{H}_{\kappa, N}}$. For $1 \leq j \leq N$, let $m_{j}$ be positive integers such that $j \leq m_{j} \leq N$. Let $\mathbf{m}=\left\{m_{1}, \ldots, m_{N}\right\}$. Define

$$
\Xi_{\mathbf{m}}(\kappa, N):=\{\mathbf{1}\} \bigcup_{n=1}^{N} \Xi_{m_{n}, n}(\kappa, N) .
$$

Theorem 5.9. For every polynomial $f \in \Pi_{N}^{d}$ of degree at most $N$ in d-variables,

$$
f=\langle f, 1\rangle_{\mathrm{H}_{\kappa, N}}+\sum_{n=1}^{N} \mathrm{D}_{n}\left(m_{n}, N\right) \sum_{|\alpha|=m_{n}} \frac{(\kappa+\mathbf{1})_{\alpha}}{\alpha !}\left\langle f, \mathrm{Q}_{\alpha, n}(\cdot ; \kappa, N)\right\rangle_{\mathrm{H}_{\kappa, N}} \mathrm{Q}_{x, n}(\alpha ; \kappa, N),
$$

and, in particular,

$$
\langle f, f\rangle_{\mathrm{H}_{\kappa, N}}=\langle f, 1\rangle_{\mathrm{H}_{\kappa, N}}^{2}+\sum_{n=1}^{N} \mathrm{D}_{n}\left(m_{n}, N\right) \sum_{|\alpha|=m_{n}} \frac{(\kappa+\mathbf{1})_{\alpha}}{\alpha !}\left\langle f, \mathrm{Q}_{\alpha, n}(\cdot ; \kappa, N)\right\rangle_{\mathrm{H}_{\kappa, N}}^{2} .
$$

Proof. The orthogonal expansion of $f \in \Pi_{N}^{d}$ in the Hahn polynomials is given by

$$
f=\sum_{n=0}^{N}\left\langle f, \mathrm{P}_{n}\left(\mathrm{H}_{\kappa, N} ; x, \cdot\right)\right\rangle_{\mathrm{H}_{\kappa, N}}
$$

Since $\mathrm{P}_{0}\left(\mathrm{H}_{\kappa, N} ; x, y\right)=1$, the the first identity follows from from (3.12), which implies (5.3). 
There is also a straightforward analogue for the Krawtchouk polynomials, which we shall not state.

Since $\left\{\mathrm{H}_{\nu}(\cdot ; \kappa, N):|\nu| \leq N\right\}$ is a mutually orthogonal basis, by writing $f$ as

$$
f_{y}(x)=\sum_{|\nu| \leq N} y_{\nu} \mathrm{H}_{\nu}(x ; \kappa, N)
$$

and using the connection (3.9), each tight frame in (5.3) corresponds to a tight frame for $\mathbb{R}^{\mathrm{n}(d, N)}$, where

$$
\mathrm{n}(d, N)=\left(\begin{array}{c}
N+d \\
d
\end{array}\right)
$$

and the frame elements can be computed directly in terms of $\mathrm{H}_{\nu}\left(x ; \kappa, m_{n}\right)$. We shall not written down these tight Euclidean frames but turn our attention to another way of constructing tight Euclidean frames using the Hahn polynomials, this time using $\mathrm{Q}_{\alpha, n}(\cdot ; \kappa, N)$ instead of $\mathrm{H}_{\nu}(\cdot ; \kappa, N)$.

This construction is based on the observation that the weight function $\mathrm{H}_{\kappa, N}$ of the Hahn polynomials becomes 1 when $\kappa=0$, so that the inner product $\langle\cdot, \cdot\rangle_{\mathrm{H}_{0, N}}$ is a constant multiple of the inner product $(\cdot, \cdot)$ of the Euclidean space $\mathbb{R}^{\mathrm{n}(d, N)}$. We use Theorem 5.9 with $\kappa=0$ and $m_{n}=N$ for $n=1,2, \ldots, N$.

Theorem 5.10. For $\alpha \in \mathbb{Z}_{N}^{d+1}$, define the vector

$$
\mathrm{q}_{\alpha, n}:=\left(\mathrm{Q}_{\alpha, n}(\beta ; 0, N): \beta \in \mathbb{Z}_{N}^{d+1}\right) \in \mathbb{R}^{\mathrm{n}(d, N)}
$$

and define $\mathrm{q}_{0}:=\sqrt{\frac{N !}{(d+1)_{N}}} \mathbf{1}$. Then the set

$$
\Xi(d, N):=\left\{\mathrm{q}_{0}\right\} \cup\left\{\mathrm{q}_{\alpha, n}: \alpha \in \mathbb{Z}_{N}^{d+1}, n=1, \ldots, N\right\}
$$

is a tight frame of $\mathbb{R}^{\mathrm{n}(d, N)}$ with $\# \Xi(d, N)=1+N \times \mathrm{n}(d, N)$ elements, that is,

$$
(x, x)=\left(x, \mathbf{q}_{0}\right)^{2}+\sum_{n=1}^{N} \sum_{|\alpha|=N}\left(x, \mathbf{q}_{\alpha, n}\right)^{2} .
$$

Proof. For $\kappa=0$, the inner product becomes

$$
\langle f, g\rangle_{\mathrm{H}_{0, N}}=\frac{N !}{(d+1)_{N}} \sum_{|\alpha|=N} f(\alpha) g(\alpha) .
$$

Since $f \in \Pi_{N}^{d}$ is uniquely determined by its values on the points $\left\{\alpha: \alpha \in \mathbb{Z}_{N}^{d+1}\right\}$, we see that $\langle f, g\rangle_{\mathrm{H}_{0, N}}=\frac{N !}{(d+1)_{N}}(\mathrm{f}, \mathrm{g})$, where $\mathrm{f}=\left(f(\beta): \beta \in \mathbb{Z}_{N}^{d+1}\right) \in \mathbb{R}^{\mathrm{n}(d, N)}$. For $m=N$ and $\kappa=0$, $\mathrm{D}_{n}(N, N)=(d+1)_{N} / N$ ! is independent of $n$. Consequently, we can write (5.3) as (5.5).

Corollary 5.11. For $n \geq 1$ and $\mathrm{q}_{\alpha, n}$ in (5.4),

$$
\left(\mathbf{q}_{\alpha, n}, \mathbf{q}_{\beta, n}\right)=\frac{\mathrm{P}_{n}\left(H_{0, N} ; \alpha, \beta\right)}{\mathrm{n}(d, N)}, \quad \alpha, \beta \in \mathbb{Z}_{N}^{d+1} .
$$

Proof. From (3.12) with $m=N$ and $\kappa=0$, it follows that

$$
\left(\mathbf{q}_{\alpha, n}, \mathbf{q}_{\beta, n}\right)=\sum_{|\gamma|=N} \mathrm{Q}_{\alpha, n}(\gamma ; 0, N) \mathbf{Q}_{\beta, n}(\gamma ; 0, N)=\frac{1}{\mathrm{D}_{n}(N, N)} \mathrm{P}_{n}\left(H_{0, N} ; \alpha, \beta\right) .
$$

Furthermore, for $\kappa=0, \mathrm{D}_{n}(N, N)=(d+1)_{N} / N !=\left(\begin{array}{c}N+d \\ N\end{array}\right)=\mathrm{n}(d, N)$. 
The elements of the tight frame $\Xi(d, N)$ are given in explicit formulas in terms of $\mathrm{Q}_{\alpha, n}(\cdot ; \kappa, N)$. In the case of $d=2$, we have

$$
\mathrm{Q}_{\alpha, n}(x ; \kappa, N)=\frac{\left[(-N)_{n}\right]^{2}(3)_{2 n}}{n !(3)_{n+N}(n+2)_{n}} \sum_{k=0}^{n} \frac{(-n)_{k}(n+2)_{k}}{\left[(-N)_{k}\right]^{2}} \mathcal{E}_{k}(\alpha, x), \quad \alpha, x \in \mathbb{Z}_{N}^{3},
$$

where $\mathcal{E}_{0}(x, y)=1$ and, for $k=1,2, \ldots, n$,

$$
\mathcal{E}_{k}(\alpha, x)=\sum_{|\gamma|=k} \frac{(-\alpha)_{\gamma}(-x)_{\alpha}}{\gamma !^{2}}=\sum_{|\gamma|=k}\left(\begin{array}{l}
\alpha \\
\gamma
\end{array}\right)\left(\begin{array}{l}
x \\
\gamma
\end{array}\right) .
$$

The vectors $q_{\alpha, n}$ are given by evaluations of these polynomials. One interesting feature is that all frame elements other than $\mathrm{q}_{0}$ have rational entries. We give the first two cases as examples. Let $e_{1}=(1,0,0), e_{2}=(0,1,0), e_{3}=(0,0,1)$.

Example 5.12. For $d=2$ and $N=1$, it follows readily that

$$
\mathrm{Q}_{\alpha, 1}(x, 0,1)=\frac{1}{3}\left(1-3 \mathcal{E}_{1}(\alpha, x)\right) \quad \text { and } \quad \mathcal{E}_{1}(\alpha, x)=\alpha_{1} x_{1}+\alpha_{2} x_{2}+\alpha_{3} x_{3} .
$$

Evaluating at the set $\left\{\alpha: \alpha \in \mathbb{Z}_{1}^{3}\right\}=\left\{e_{1}, e_{2}, e_{3}\right\}$ shows that the column of the matrix

$$
\Xi(2,1)=\left[\begin{array}{cccc}
\frac{1}{\sqrt{3}} & \frac{2}{3} & -\frac{1}{3} & -\frac{1}{3} \\
\frac{1}{\sqrt{3}} & -\frac{1}{3} & \frac{2}{3} & -\frac{1}{3} \\
\frac{1}{\sqrt{3}} & -\frac{1}{3} & -\frac{1}{3} & \frac{2}{3}
\end{array}\right]
$$

form a tight frame in $\mathbb{R}^{3}$ with 4 vectors.

Example 5.13. For $d=2$ and $N=2, \mathbb{Z}_{2}^{3}=\left\{2 e_{1}, 2 e_{2}, 2 e_{3}, e_{1}+e_{2}, e_{2}+e_{3}, e_{+} e_{1}\right\}$. We have $\mathbf{q}_{0}=\frac{1}{\sqrt{6}}(1,1,1,1,1,1)$. For $n \geq 1$, there are two cases. For $\alpha \in \mathbb{Z}_{2}^{3}$ and $n=1$, we obtain

$$
\mathrm{Q}_{\alpha, 1}(x, 0,1)=-\frac{4}{15}\left(1-\frac{3}{4} \mathcal{E}_{1}(\alpha, x)\right), \quad \mathcal{E}_{1}\left(2 e_{i}, x\right)=2 x_{i}, \quad \mathcal{E}_{1}\left(e_{i}+e_{j}\right)=x_{i}+x_{j},
$$

and for $\alpha \in \mathbb{Z}_{2}^{3}$ and $n=2$, we obtain

$$
\mathrm{Q}_{\alpha, 2}(x, 0,1)=\frac{1}{10}\left(1-2 \mathcal{E}_{1}(\alpha, x)+10 \mathcal{E}_{2}(\alpha, x)\right),
$$

where $\mathcal{E}_{1}(\alpha, x)$ is as before and

$$
\mathcal{E}_{2}\left(2 e_{i}, x\right)=\frac{1}{2} x_{i}\left(x_{i}-1\right), \quad \mathcal{E}_{2}\left(2 e_{i}+e_{j}, x\right)=x_{1} x_{2} .
$$

Evaluating these two functions at elements in $\mathbb{Z}_{2}^{3}$, we obtain $\mathbf{q}_{\alpha, 1}$ and $\mathbf{q}_{\alpha, 2}$. The set $\Xi(2,2)$ consists of columns of the matrix

$$
\left[\begin{array}{ccccccccccccc}
\frac{1}{\sqrt{6}} & -\frac{3}{10} & -\frac{3}{10} & \frac{1}{10} & \frac{1}{10} & \frac{3}{10} & \frac{1}{10} & -\frac{4}{15} & \frac{2}{15} & \frac{2}{15} & -\frac{4}{15} & -\frac{4}{15} & \frac{8}{15} \\
\frac{1}{\sqrt{6}} & -\frac{3}{10} & \frac{1}{10} & -\frac{3}{10} & \frac{1}{10} & \frac{1}{10} & \frac{3}{10} & \frac{2}{15} & -\frac{4}{15} & \frac{2}{15} & -\frac{4}{15} & \frac{8}{15} & -\frac{4}{15} \\
\frac{1}{\sqrt{6}} & \frac{1}{10} & -\frac{3}{10} & -\frac{3}{10} & \frac{3}{10} & \frac{1}{10} & \frac{1}{10} & \frac{2}{15} & \frac{2}{15} & -\frac{4}{15} & \frac{8}{15} & -\frac{4}{15} & -\frac{4}{15} \\
\frac{1}{\sqrt{6}} & \frac{7}{10} & -\frac{1}{10} & -\frac{1}{10} & \frac{1}{10} & -\frac{3}{10} & -\frac{3}{10} & -\frac{1}{15} & -\frac{1}{15} & \frac{2}{15} & -\frac{4}{15} & \frac{2}{15} & \frac{2}{15} \\
\frac{1}{\sqrt{6}} & -\frac{1}{10} & \frac{7}{10} & -\frac{1}{10} & -\frac{3}{10} & -\frac{3}{10} & \frac{1}{10} & -\frac{1}{15} & \frac{2}{15} & -\frac{1}{15} & \frac{2}{15} & -\frac{4}{15} & \frac{2}{15} \\
\frac{1}{\sqrt{6}} & -\frac{1}{10} & -\frac{1}{10} & \frac{7}{10} & -\frac{3}{10} & \frac{1}{10} & -\frac{3}{10} & \frac{2}{15} & -\frac{1}{15} & -\frac{1}{15} & \frac{2}{15} & \frac{2}{15} & -\frac{4}{15}
\end{array}\right]
$$

and these column vectors form a tight frame in $\mathbb{R}^{6}$ with 13 vectors. Apart from the first vector $\mathrm{q}_{0}$, all other vectors have rational entries.

For $d=2$ and each $N=1,2, \ldots$, the identity (5.5) gives a tight frame of $\mathbb{R}^{\mathrm{n}(2, N)}$ with $M:=N \times \mathrm{n}(2, N)+1$ vectors. The next one is a tight frame of $\mathbb{R}^{10}$ with 31 vectors. Except $\mathrm{q}_{0}$, all other frame elements in a tight frame of this family have rational entries. 


\section{Acknowledgements}

The work was supported in part by NSF Grant DMS-1106113.

\section{References}

[1] Bachoc C., Ehler M., Tight p-fusion frames, Appl. Comput. Harmon. Anal. 35 (2013), 1-15, arXiv:1201.1798.

[2] Benedetto J.J., Fickus M., Finite normalized tight frames, Adv. Comput. Math. 18 (2003), 357-385.

[3] Casazza P.G., Custom building finite frames, in Wavelets, frames and operator theory, Contemp. Math., Vol. 345, Amer. Math. Soc., Providence, RI, 2004, 61-86.

[4] Casazza P.G., Kutyniok G., Philipp F., Introduction to finite frame theory, in Finite Frames, Appl. Numer. Harmon. Anal., Birkhäuser/Springer, New York, 2013, 1-53.

[5] Diaconis D., Griffiths R., An introduction to multivariate Krawtchouk polynomials and their applications, arXiv:1309.0112.

[6] Dunkl C.F., Xu Y., Orthogonal polynomials of several variables, Encyclopedia of Mathematics and its Applications, Vol. 81, Cambridge University Press, Cambridge, 2001.

[7] Griffiths R.C., Spanò D., Multivariate Jacobi and Laguerre polynomials, infinite-dimensional extensions, and their probabilistic connections with multivariate Hahn and Meixner polynomials, Bernoulli 17 (2011), 1095-1125, arXiv:0809.1431.

[8] Griffiths R.C., Spanò D., Orthogonal polynomial kernels and canonical correlations for Dirichlet measures, Bernoulli 19 (2013), 548-598, arXiv:1003.5131.

[9] Heil C., What is ... a frame?, Notices Amer. Math. Soc. 60 (2013), 748-750.

[10] Iliev P., Xu Y., Discrete orthogonal polynomials and difference equations of several variables, Adv. Math. 212 (2007), 1-36, math.CA/0508039.

[11] Karlin S., McGregor J., Linear growth models with many types and multidimensional Hahn polynomials, in Theory and Application of Special Functions (Proc. Advanced Sem., Math. Res. Center, Univ. Wisconsin, Madison, Wis., 1975), Editor R.A. Askey, Academic Press, New York, 1975, 261-288.

[12] Rosengren H., Multivariable orthogonal polynomials and coupling coefficients for discrete series representations, SIAM J. Math. Anal. 30 (1999), 232-272.

[13] Tratnik M.V., Multivariable biorthogonal Hahn polynomials, J. Math. Phys. 30 (1989), 627-634.

[14] Tratnik M.V., Multivariable Meixner, Krawtchouk, and Meixner-Pollaczek polynomials, J. Math. Phys. 30 (1989), 2740-2749.

[15] Tratnik M.V., Some multivariable orthogonal polynomials of the Askey tableau-discrete families, J. Math. Phys. 32 (1991), 2337-2342.

[16] Waldron S., On the Bernstein-Bézier form of Jacobi polynomials on a simplex, J. Approx. Theory 140 (2006), 86-99.

[17] Waldron S., Continuous and discrete tight frames of orthogonal polynomials for a radially symmetric weight, Constr. Approx. 30 (2009), 33-52.

[18] Xu Y., Monomial orthogonal polynomials of several variables, J. Approx. Theory 133 (2005), 1-37.

[19] Xu Y., Hahn, Jacobi, and Krawtchouk polynomials of several variables, arXiv:1309.1510. 